
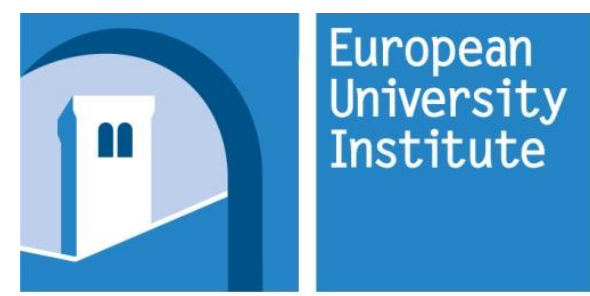

ROBERT

SCHUMAN

CENTRE FOR

ADVANCED

STUDIES

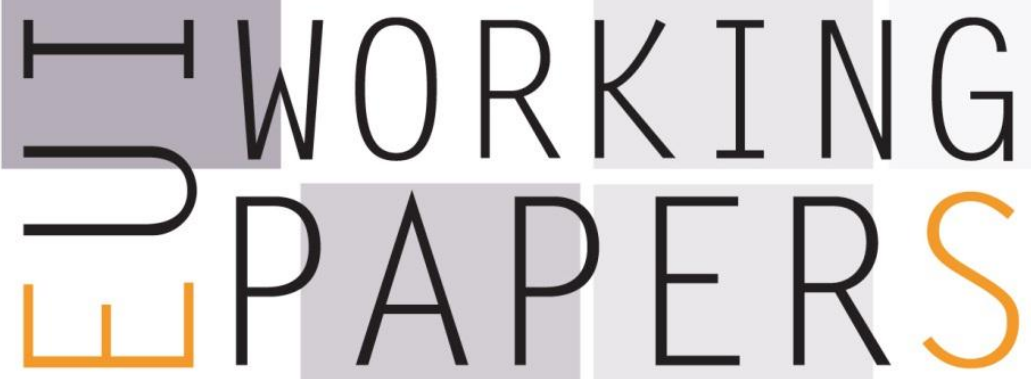

RSCAS 2013/57

Robert Schuman Centre for Advanced Studies

Network Neutrality, Access to Content and Online Advertising

Anna D'Annunzio and Antonio Russo 

European University Institute

Robert Schuman Centre for Advanced Studies

Network Neutrality, Access to Content and Online Advertising

Anna D’Annunzio and Antonio Russo

EUI Working Paper RSCAS 2013/57 
This text may be downloaded only for personal research purposes. Additional reproduction for other purposes, whether in hard copies or electronically, requires the consent of the author(s), editor(s). If cited or quoted, reference should be made to the full name of the author(s), editor(s), the title, the working paper, or other series, the year and the publisher.

ISSN 1028-3625

(c) Anna D’Annunzio and Antonio Russo, 2013

Printed in Italy, July 2013

European University Institute

Badia Fiesolana

I - 50014 San Domenico di Fiesole (FI)

Italy

www.eui.eu/RSCAS/Publications/

www.eui.eu

cadmus.eui.eu 


\section{Robert Schuman Centre for Advanced Studies}

The Robert Schuman Centre for Advanced Studies (RSCAS), created in 1992 and directed by Stefano Bartolini since September 2006, aims to develop inter-disciplinary and comparative research and to promote work on the major issues facing the process of integration and European society.

The Centre is home to a large post-doctoral programme and hosts major research programmes and projects, and a range of working groups and ad hoc initiatives. The research agenda is organised around a set of core themes and is continuously evolving, reflecting the changing agenda of European integration and the expanding membership of the European Union.

Details of the research of the Centre can be found on:

http://www.eui.eu/RSCAS/Research/

Research publications take the form of Working Papers, Policy Papers, Distinguished Lectures and books. Most of these are also available on the RSCAS website:

http://www.eui.eu/RSCAS/Publications/

The EUI and the RSCAS are not responsible for the opinion expressed by the author(s). 



\title{
Network Neutrality, Access to Content and Online Advertising*
}

\author{
Anna D’Annunzio ${ }^{\dagger} \quad$ Antonio Russo ${ }^{\ddagger}$
}

June 2013

\begin{abstract}
We investigate possible effects of network neutrality regulation on the distribution of content in the Internet. We model a two-sided market, where consumers and advertisers interact through Content Providers (CPs), and CPs and consumers through Internet Service Providers (ISPs). Multiple impressions of an ad on a consumer are partially wasteful. Thus, equilibrium ad rates decrease with the number of CPs consumers can browse. Under network neutrality, CPs can connect to any ISP for free, while in the unregulated regime they have to pay a (non-discriminatory) access fee set by the ISP. We show that universal distribution of content is always an equilibrium with net neutrality regulation. Instead, in the unregulated regime, ISPs can use access fees to rule out universal distribution when it is not profitable, i.e. when repeated impressions of an ad rapidly lose value and consumers care for content availability to a small extent. We also find that the unregulated regime is never superior to net neutrality from a welfare point of view. Consumer and advertiser surplus are weakly higher under net neutrality. ISPs are unambiguously better off in the unregulated regime, while CPs are unambiguously worse off.
\end{abstract}

Keywords: network neutrality, two-sided markets, Internet, advertising, fragmentation

JEL Classification: L1, D43, L13, L51

\footnotetext{
${ }^{*}$ We thank Bruno Jullien for his important advice. We also thank Jacques Crémer, Carole Haritchabalet, Yassine Lefouili, Pierfrancesco Reverberi, Wilfried Sand-Zantman and Paul Seabright for helpful comments and discussions. All errors are ours.

†Toulouse School of Economics (GREMAQ) and University of Rome "Sapienza" (DIAG)

${ }^{\ddagger}$ RSCAS, European University Institute
} 


\section{Introduction}

Traditionally, the Internet has been characterized by the net neutrality principle. This principle refers, in a nutshell, to the fact that all Internet traffic should be treated equally and has various practical implications. In particular, it implies a zero-price and a non-discrimination rule (Schuett, 2010). The former refers to the fact that Internet Service Providers (hereafter, ISPs) should not collect fees from Content Providers (hereafter, CPs) to deliver (or "terminate") contents to final users, while the latter refers to the fact that ISPs should not provide different quality of service to different CPs. Presently, the extent to which net neutrality should be codified in formal regulation is the subject of a very important policy debate. Opponents assert that termination fees and traffic discrimination are vital to ensure a more efficient use of bandwidth and strengthen incentives for investment in network infrastructure. Proponents argue instead that net neutrality regulation is necessary to preserve plurality on the Internet, alongside incentives for innovation and creation of content (Kramer et al., 2012).

The above issues have received considerable attention in previous economic literature (which we review in Section 2 below). There is however another aspect of net neutrality that has been much less scrutinized thus far. Namely, the implications of (relaxing) a zero price rule for Internet fragmentation and for competition on the online advertising market. While pricing and traffic management can, in principle, improve efficiency, there are concerns that they could also lead to a fragmented Internet, with portions of consumers not being able to access content available to others (Lee and Wu, 2009, Werbach, 2009).

Policymakers are clearly sensitive to the issue. In the US, the FCC adopted a policy statement adhering to the principle that "consumers are entitled to access the lawful Internet content of their choice" (FCC, 2005). European Commission Vice-President Neelie Kroes stated that "any content or application that is legal and which does not cause undue congestion or otherwise harm other users or network integrity should be fully accessible" (see http://edri.org/node/3281). Furthermore, Dutch MEP Marietje Schaake recently expressed concerns that "In the absence of regulation, internet providers could block access to certain news or entertainment sites because of their financial interests". ${ }^{1}$ As a matter of fact, the Netherlands are among the countries that formally adopted legislation forbidding ISPs from charging termination fees to content providers. Several other governments are considering doing the same.

Our objective in this paper is to shed some light on these issues. To keep the analysis as simple and transparent as possible, we consider a model with two CPs and analyze both the case of a monopolist ISP and of two competing ISPs. An ISP is a platform connecting

\footnotetext{
${ }^{1}$ See EUObserver. com, "Brussels to table net neutrality rules". Retrieved June 2013.
} 
consumers to CPs and a CP is a platform selling consumers' attention to advertisers. Consumers pay the ISP for connection to the Internet and browse content free of charge. We study two different regulatory regimes: under net neutrality, a zero-price rule is enforced and CPs can connect to ISPs for free. In the unregulated regime, instead, ISPs make access to their users conditional on the payment of an access fee. ${ }^{2}$

It is well recognized that the Internet is a two-sided market bringing together consumers and advertisers. Yet, to the best of our knowledge, previous literature on net neutrality has never explicitly modeled the advertising side. In contrast, we model revenue collected by CPs as arising from equilibrium outcomes in that market. Our model accounts for the stylized fact that the marginal value of impressing a consumer with an ad decreases with the number of times she is exposed to it. For instance, an ad that reaches a consumer that is already informed about a product could be (at least partially) wasted, since she could elicit no more information from it. ${ }^{3}$ As suggested by Athey et al. (2012), the fact that consumers consult several contents in a short time frame (which is common on the Internet) means that advertisers placing ads on multiple CPs run the risk that their impressions are viewed by the same consumer multiple times. Consequently, the willingness to pay for advertising slots by multi-homing advertisers diminishes when audiences overlap. ${ }^{4}$ This implies that a CP may prefer a small audience, that can browse few other contents, to a large one that browses a wide set of contents. Indeed, in our model, when impressing an ad on a consumer after the first time is much less valuable than doing it for the first time, competition among CPs strongly reduces the profitability of ad spaces. On the contrary, when repeated ad impressions lose little value, the impact of competition on advertising profits is limited.

There is evidence that, while advertisers place increasingly large quantities of ads online, competition keeps advertising rates low. ${ }^{5}$ As long as competition to attract advertisers reduces profits, one would expect CPs to try to soften it by targeting different consumers. However, when connection to ISPs is free for CPs this may prove difficult to sustain. ${ }^{6}$ This

\footnotetext{
${ }^{2}$ We disregard service tiering and quality discrimination for simplicity. One could however see exclusion from an ISP's network in our model as resulting from strong degradation of service for CPs that do not acquire access to the high quality tier.

${ }^{3}$ See, for example, Calvano and Jullien (2011), Anderson et al. (2011), Athey et al. (2012) and Ambrus and Reisinger (2006).

${ }^{4}$ As a response, Internet platforms introduce tracking technologies that reduce within-outlet waste. However, if cross-outlet tracking is imperfect (or, in the extreme, absent as we assume) the problem is not solved.

${ }^{5}$ Todd Haskell, vice-president of advertising for the New York Times, stated that rates for online-video ads have recently not been increasing though publishers have more demand than supply. He also stated that publishers may seek differentiation from competitors as a way to "avoid the downward commoditized price positioning" of ad slots (from "If Media's Future Is Online, Where Are the Profits?" - WSJ.com, retrieved June 2013).

${ }^{6}$ Taylor (2012) argues that "Advertisers [...] value exclusive access to consumers' attention" and "publishers typically have no direct influence over their rivals' advertising business."
} 
suggests that there may be an important link between network neutrality, Internet fragmentation and competition on the advertising market. Indeed, we show that, by strategically setting non-discriminatory access fees, ISPs may fragment the Internet. The rationale for doing so, we argue, is to increase the power of the distributed CPs on the advertising market. This, in turn, increases the profits that ISPs can extract from them.

The results we obtain run as follows. In the net neutrality regime, ISPs have a very passive role. If a single ISP is active on the market, all CPs connect to it in equilibrium. If two ISPs operate in the market, there is always an equilibrium where all CPs access both ISPs (to which we refer as "universal connection"). When the profitability of competitively priced ad spaces is too low, there also exists an equilibrium in which each CP joins a different ISP (to which we refer as "total fragmentation"). This is, in particular, the case when repeated impressions of an ad on the same consumers rapidly lose value. We also find that the extent to which consumers value contents and their complementarity play quite a counterintuitive role in the net neutrality regime. Both reduce (all else given) the likelihood of universal connection and increase that of total fragmentation. This crucially depends on the fact that advertising rates decrease with overlapping viewerships.

In the unregulated regime, CPs can access an ISP's consumers only by paying a fee. This significantly changes the forces shaping the network configuration. Since they can recover CPs profits via the access fee, ISPs behave as editors, caring about the profitability of the content they carry. Hence, when competition strongly reduces ad rates, ISPs have an incentive to use access fees to discard universal connection. If a single ISP is active on the market, the equilibrium is such that both CPs access the network when competitive ad rates are high enough, while one is excluded when they are small, hence when repeated ad impressions have little value. ${ }^{7}$ With a duopoly of ISPs, we obtain that when the impact of competition on advertising profits is limited, the equilibrium entails universal distribution. Otherwise, total fragmentation is the unique equilibrium. In the unregulated regime, content value and complementarity play a more intuitive role than under net neutrality. Both reduce the likelihood (all else given) of total fragmentation, fostering competition among ISPs to attract content. However, complementarity does not affect the likelihood of universal connection.

When comparing the two regulatory regimes, our results suggest that the absence of net neutrality regulation makes universal distribution of contents less likely. More precisely, conditions for universal connection to be the unique equilibrium are always weaker with net neutrality than in its absence. Moreover, with net neutrality, universal connection is always an equilibrium, even if each CP would make higher profits with a segmented network, being exclusively distributed by a different ISP. In contrast, in the unregulated regime, the strategic

\footnotetext{
${ }^{7}$ With a monopolist ISP, an equilibrium always exists and is unique.
} 
use of access fees by ISPs can impede universal connection. Indeed, when competition among CPs strongly impacts their profitability, total fragmentation is the unique equilibrium in the unregulated regime and universal connection is unattainable. However, a change in the regulatory regime does not necessarily imply a change in the network configuration. First, when the impact of competition on advertising profits is small, universal distribution is the only equilibrium, even without net neutrality regulation. Second, total fragmentation can take place even if net neutrality is maintained. This is the case when competition among CPs strongly reduces their profitability as advertising outlets. It follows that, although net neutrality certainly helps preserving universal access to online content, the extent to which repeated ad impressions lose value plays an important role in shaping the network configuration, regardless of the regulatory regime.

By influencing the market configuration that emerges in equilibrium, the regulatory regime may strongly affect social welfare. Since it maximizes gross surplus at both ends of the market (i.e. for consumers and advertisers), universal distribution is the most desirable configuration for society as a whole. Also consumer surplus is maximized under universal distribution, since consumers enjoy more contents and transportation costs are minimized. Hence, net neutrality (weakly) maximizes, consumers and social welfare. Finally, without net neutrality, ISPs are unambiguously better off, while CPs are unambiguously worse off.

The remainder of this paper is organized as follows. Section 2 reviews the relevant literature. Section 3 presents the model, which is solved in Section 4 for the monopoly ISPs and Section 5 for duopoly ISPs. Section 6 concludes. Part of the proofs are relegated to the Appendix.

\section{Literature}

There is a wide debate on net neutrality, involving lawyers, engineers and economists, that has only recently been formally analyzed from an economic perspective. ${ }^{8}$ Departing from network neutrality could allow service tiering by ISPs, i.e., provision to CPs of different network qualities for different prices. This is often advocated as a way to allow ISPs to better deal with congestion. Hermalin and Katz (2007) study the desirability of a discriminatory regime. When net neutrality is imposed, an ISP is forced to offer a unique quality to all content providers. When an ISP is free to discriminate, more attractive contents purchase higher quality. Compared to the unregulated model, welfare can increase or decrease under

\footnotetext{
${ }^{8}$ See Lee and $\mathrm{Wu}(2009)$ for a discussion on the economic issues concerning net neutrality. See Schuett (2010) and Krämer et al. (2012) for a review of the literature.
} 
net neutrality. The authors argue that these effects are often negative. ${ }^{9}$ Choi and Kim (2010) show that investment incentives by a monopolist ISP can be either higher or lower in the discriminatory regime than with net neutrality, since capacity expansion decreases the value of the scarce resource, i.e. the fast line. ${ }^{10}$ Bourreau, Kourandi and Valletti (2012) study a similar issue in a model with two competing ISPs. Under discrimination, ISPs have larger investment incentives, more content providers are active and there is less congestion. Hence, the discriminatory regime is welfare superior to the net neutrality regime. Economides and Hermalin (2012) show a result that we also find: the preferred configuration from a welfare point of view is the one where more contents are delivered to consumers. Differently from Bourreau, Kourandi and Valletti (2012), departing from net neutrality through a tiering service can either increase or decrease total content sent, and then welfare. ${ }^{11}$ In the long run, they also show, contrary to Choi and Kim (2010), that departing from net neutrality unambiguously increases the incentives of the ISP to invest in the bandwidth. However, the net welfare effect, taking into account dynamic and static effects, is not a priori clear. The authors show that, when consumer surplus weighs a lot on total welfare, net neutrality is welfare-enhancing.

These papers focus on service tiering, while we consider net neutrality as a zero price rule on the content side. In our paper, without net neutrality, ISPs are allowed to impose fees to CPs which want to reach their consumers. Economides and Tag (2012) use this definition of net neutrality and concentrate on the pricing issue linked to the two-sidedness of the market that could arise due to a departure from net neutrality. Considering a duopoly of ISPs, they find that, when content providers value consumers more than consumers value contents, welfare increases under net neutrality, since the platform internalizes cross-group externalities. On the contrary, consumer surplus always decreases, since competition for consumers is less intense under net neutrality than when cross-group externalities are taken into account by the platform in the two-sided model under no regulation. Also Musacchio, Schwartz and Walrand (2009) analyze a similar issue in a model where ISPs and CPs can

\footnotetext{
${ }^{9}$ Choi, Jeon and Kim (2012) study a similar issue with two interconnected ISPs. They show that the advantage of net neutrality regulation crucially depends on the business model of the content providers, since this affects the quality level provided under different regimes.

${ }^{10}$ Choi and Kim (2010) also study the incentives of CPs to invest in content quality: the incentive to invest of the high-speed content depends on its ability to exploit the fast line and on its bargaining power, while the rival content always invests less under the discriminatory regime.

${ }^{11}$ Both Bourreau, Kourandi and Valletti (2012) and Economides and Hermalin (2012) model a continuum of CPs with a different sensitivity to congestion. Economides and Hermalin (2012) also take into account the elasticity of consumers' demand with respect to the transmission time. They conclude that welfare increases when the delay times are shorter for contents with greater elasticities of demand with respect to transmission time. Remark that more time sensitive contents need not to be the contents with the highest elasticity. Thus, in their model, prioritizing these contents could entail a loss of welfare.
} 
also invest. They find that net neutrality can be either welfare superior or inferior to the unregulated regime.

As mentioned in the Introduction, previous papers on net neutrality model profitability of content providers as an exogenous parameter. In contrast, we derive it endogenously, as a result of CPs' competition on the advertising market. Moreover, these models do not consider the segmentation issue that could arise when net neutrality is removed (see Lee and $\mathrm{Wu}, 2009)$. Indeed, a CP can sell the attention of exclusive consumers to advertisers for a higher price than the attention of non-exclusive consumers (see Ambrus and Reisinger, 2006, Calvano and Jullien, 2011, and Anderson, Foros and Kind, 2011). As a consequence, some contents could be accessible from an ISP but not from the other. ${ }^{12}$

In an independently developed paper, Kourandi et al. (2013) also consider the fragmentation issue. Although the main question they investigate is essentially the same as we do, there are some important differences between the two approaches and the results we found. First of all, their main focus is on exclusive contracts among ISPs and CPs. In contrast, we study how fragmentation can result from allowing ISPs to set simple non-discriminatory access fees. Kourandi et al. also include a termination fee, but they assume it is the same for all ISPs and exogenous. Thus, our approach investigates the link between pricing regulation and Internet fragmentation in a different (and, in a sense, more direct) way. We argue that, short of making use of exclusivity contracts, ISPs can induce a given configuration of the network by strategically setting the access fee. ${ }^{13}$ Secondly, in our model, once ISPs have set the access fees, connection decisions are made simultaneously by CPs. In Kourandi et al., ISPs contract sequentially with CPs for exclusivity. Third, the interpretation of net neutrality slightly differs among the two papers. In our paper, net neutrality means that access fees are restricted to zero. In Kourandi et al. (2013) net neutrality entails either zero termination fees or zero termination fees and zero-transfers for exclusive contracts. Finally, we explicitly model the advertisers side of the market. Kourandi et al. assume that the revenue per impression for exclusive consumers is higher than for non-exclusive ones. However, they do not model the forces behind this equilibrium outcome.

The differences mentioned above are relevant for the results. Differently fromKourandi et $a l$., we find that universal connection is always an equilibrium under net neutrality. The difference comes from the different way in which the negotiation stage is modeled under net neutrality. More precisely, we assume that with net neutrality ISPs have no instrument to

\footnotetext{
${ }^{12}$ Bourreau, Kourandi and Valletti (2012), Economides and Hermalin (2012) and Hermalin and Katz (2007) evaluate how many content providers are active in equilibrium under different regulatory regimes.

${ }^{13}$ Kourandi et al. (2013) also restrict the set of possible network configurations: they rule out cases in which both CPs join only one ISP. We instead consider this case: even if it never arises in equilibrium, it does affect the equilibrium outcomes in the unregulated regime.
} 
influence CPs' connections decisions. On the contrary, Kourandi et al. use a sequential negotiation for exclusive contracts, and a CP that has signed an exclusivity contract with an ISP cannot connect to the rival, even with net neutrality. Furthermore, findings concerning the role of content value and complementarity are quite divergent: for instance, they find that complementarity makes total fragmentation (resp. universal connection) more (less) likely in the unregulated regime. We find the opposite. Finally, Kourandi et al. find, unlike us, that universal distribution and net neutrality are not necessarily socially desirable. This difference is due to the fact that we take explicitly into consideration advertiser surplus when computing total welfare.

\section{The Model}

\subsection{Setup}

We consider a setting with two Internet Service Providers (ISPs), indexed by $i=A, B$, and two Content Providers (CPs), indexed by $j=0,1$. An ISP is a platform connecting consumers to $\mathrm{CPs}$, and a $\mathrm{CP}$ is a platform connecting consumers to advertisers. Obviously, a CP needs to be connected to at least one ISP in order to be able to reach consumers. Moreover, each CP reaches only consumers of the ISP it is connected to. ISPs and CPs are independent firms.

We compare connection choices made by firms in two alternative regulatory regimes: one in which network neutrality regulation is in place and one in which it is not, referred to as the unregulated regime. In the former case, ISPs have to grant access to their network to all CPs for free: we consider net neutrality as a zero-price rule for CPs (see, e.g., Schuett, 2010). In the latter, ISPs can impose an access fee to CPs.

Internet Service Providers. ISPs are located at the extremes of a Hotelling line: ISP $i=A$ is located at point 0 and ISP $i=B$ at point 1 . ISP $i$ sets a subscription fee $a_{i}$ for consumers who want to join its network. In the unregulated regime, ISP $i$ also sets an access fee $F_{i}$ for CPs that want to reach its consumers. $F_{i}$ is exclusionary: a CP can access ISP $i$ 's consumer base only if agrees to pay it. ${ }^{14}$ In the net neutrality regime, CPs can freely access both ISPs, hence $F_{i}=0$. All fees are non-discriminatory and non-contingent. The profit function of ISP $i$ is

$$
\pi_{i}=a_{i} q_{i}+\sum_{j=0,1} F_{i} I_{i j} \quad i=A, B
$$

\footnotetext{
${ }^{14}$ There is no a priori restriction on the sign of access fees. However, they always turn out to be positive in equilibrium, as we show below.
} 
where $q_{i}$ is the number of consumers connected to ISP $i$ and $I_{i j}$ is an indicator function, such that $I_{i j}=1$ if CP $j$ acquires access to ISP $i$ 's consumers and $I_{i j}=0$ otherwise. More details on this function are provided below.

Consumers. There is a large mass (normalized to one) of consumers uniformly distributed on the interval $[0,1]$. Consumers connect to one and only one ISP. ${ }^{15}$ The utility $U_{i}$ a consumer located in $x \in[0,1]$ gets from connecting to ISP $i$ is

$$
U_{i}(x)=Z+\Delta_{i}-t\left|x-l_{i}\right|-a_{i} \quad i=A, B
$$

where $Z$ is the gross surplus from accessing the Internet, assumed large enough that all consumers connect to an ISP. $t$ is the transportation cost on the Hotelling line, i.e. the disutility for consumer $x$ from not consuming its preferred specification of the product. $l_{i}$ represents the ISP's position on the Hotelling line, so $l_{A}=0$ and $l_{B}=1 . \Delta_{i}$ represents the utility consumers get from browsing contents available on ISP $i$. We assume the following

$$
\Delta_{i}= \begin{cases}0 & \text { if no CP is available on ISP } i \\ \delta & \text { if one CP is available on ISP } i \\ \delta(1+\gamma) & \text { if both are available }\end{cases}
$$

with $\delta, \gamma \geq 0$. The term $\delta$ represents the utility consumers obtain from accessing a single content, while $\delta \gamma$ captures the extra utility of accessing an additional one. Hence, $\delta$ can be interpreted as the value consumers attribute to content availability in absolute terms, while $\gamma$ represents the degree of complementarity among the two contents. We do not assume any a priori difference among contents and we allow them to be either substitutes $(\gamma<1)$, complements $(\gamma>1)$ or independent $(\gamma=1)$. A priori, all of these cases can occur in reality. For example, two newspaper websites could be substitute contents. Contents such as VoIP and email services could instead be complementary. We do not endogenize consumers' demand for contents and simply assume that each consumer visits all available contents once. ${ }^{16}$ We also ignore any (dis)utility from ads. We assume that the market is covered and that the demand for each ISP is positive, i.e. $\sum_{i} q_{i}=1$ and $q_{i}>0 \forall i{ }^{17}$

\footnotetext{
${ }^{15}$ We assume consumers subscribe to just one platform to access the Internet, as is currently the most widespread practice. It is however conceivable that consumers might multi-home if platforms provide access to different contents. Our results still hold if the number of multi-homing consumers is not too large.

${ }^{16}$ More precisely, we assume that each consumer visits all available contents a given number of times $N$, that we assume without loss of generality to be equal to 1. This same assumption is used by, e.g., Bourreau et al. (2012), Choi et al. (2012) and Krämer and Weiwiorra (2012).

${ }^{17}$ The assumption of covered market is instead dropped in the case of a monopolist ISP.
} 
Net consumer surplus $(C S)$ can be computed as

$$
C S=\int_{0}^{q_{A}}\left(Z+\Delta_{A}-t x-a_{A}\right) d x+\int_{q_{A}}^{1}\left(Z+\Delta_{B}-t(1-x)-a_{B}\right) d x
$$

that can be written as

$$
C S=Z+\sum_{i=A, B}\left(\Delta_{i}-a_{i}\right) q_{i}+\frac{t}{2}-t q_{B}-t q_{A}^{2}
$$

Content Providers. Each CP provides free contents to consumers but charges advertisers that place ads on its platform. A CP $j$ charges a per-impression price $p_{j}$ to an advertiser if and only if a consumer is exposed to the ad while browsing its content. We assume there is no cost of providing ad spaces and that CPs set a uniform price for advertisers. We assume each visit by a consumer on a given content brings to an impression. Hence, the volume of impressions for an ad put on $\mathrm{CP} j$ is equal to $q_{j}$, which is the number of consumers connected to the ISPs distributing $j$ 's content, i.e.

$$
q_{j}=\sum_{i=A, B} q_{i} I_{i j} \quad j=0,1
$$

The profit of $\mathrm{CP} j$ is

$$
\pi_{j}=d_{j} p_{j} q_{j}-\sum_{i=A, B} F_{i} I_{i j} \quad j=0,1
$$

where $d_{j}$ is the number of advertisers on $\mathrm{CP} j$.

A CP can connect to either no ISP, only one or both. CPs make connection decisions by comparing profits under all network configurations, taking as given the couple $\left(F_{A}, F_{B}\right)$ and the decision of the rival $\mathrm{CP}$. We assume that, if a $\mathrm{CP}$ is indifferent between joining or not an ISP, it joins it. A given network configuration arises at equilibrium when no CP finds a profitable unilateral deviation.

Advertisers. There is a mass of size one of advertisers interested in reaching consumers by means of CPs. Their willingness to pay for ad slots crucially depends on the probability that consumers will be impressed with their ads. In turn, this depends on the number of outlets an advertiser can use to reach a given consumer. $V^{\prime}$ denotes the gross surplus (i.e. the increase in the expected value of sales due to the consumer seeing the ad) produced by reaching a consumer through a single CP. We denote by $V^{\prime \prime}$ the additional gross surplus produced by reaching the same consumer through an additional content.

Recent literature on online advertising suggests that ads seen multiple times are par- 
tially wasteful (Calvano and Jullien, 2011), since they reach already informed consumers and are therefore squandered together with their attention. ${ }^{18}$ Therefore, we make the following assumption:

$$
V^{\prime} \geq V^{\prime \prime} \geq 0
$$

Let us now provide a micro foundation for this assumption. Assume that each advertiser is a monopolist producer of a differentiated good of quality $k$. Per each good, there exists a fraction $\alpha$ of consumers with willingness-to-pay equal to $k>0$, while the rest has valuation zero. All consumers are impressed with all ads placed on a content when visiting it. Since each producer has monopoly power, it imposes a price equal to $k$, that is, a price that extracts all consumer surplus. When (and only when) a consumer with a positive valuation for the good is informed about it, she buys it. The consumer is informed when she is impressed with an ad she pays attention to. There is a probability $\beta \in[0,1]$ that a consumer pays attention to an ad when impressed. ${ }^{19}$ Hence, the expected value of impressing a consumer with an ad for the first time is:

$$
V^{\prime}=k \alpha \beta
$$

Since consumers visit all available content once (by assumption), $V^{\prime}$ is the value of reaching the consumer through a single content. A consumer can be impressed by the same ad for a second time while visiting another content. The expected value of impressing the consumer for a second time is

$$
V^{\prime \prime}=k \alpha(1-\beta) \beta
$$

that is, the expected value given that the consumer did not pay attention when impressed for the first time (note that an impression which the consumer has already paid attention to has no value since the consumer is already informed). Clearly, $V^{\prime} \geq V^{\prime \prime}$. In the following, we will use the reduced form for the gross utility of an advertising slot for advertisers.

A CP $j$ sells ads at a uniform per-impression price $p_{j} .{ }^{20}$ Advertisers can buy ad spaces from none, one or both CPs. We assume that, if advertisers are indifferent between joining a CP or not, they join it. Suppose an advertiser multi-homes, buying ad space from both content providers. Its total surplus would be

$$
V^{\prime} \bigcup_{j=0,1} q_{j}+V^{\prime \prime} \bigcap_{j=0,1} q_{j}-\sum_{j=0,1} p_{j} q_{j}
$$

\footnotetext{
${ }^{18}$ Athey et al. (2012) estimate that more than two thirds of the ads in large campaigns are wasted, hitting the same receivers more than 10 times.

${ }^{19}$ We here follow Anderson and Coate (2005) and Ambrus and Reisinger (2006).

${ }^{20} \mathrm{We}$ assume that there is no across-outlet tracking. Hence, a CP has no knowledge on whether a given consumer has already been impressed with the ad while browsing the rival's content or not. This means that prices $p_{j}$ cannot be discriminated accordingly.
} 
where $\bigcup_{j=0,1} q_{j}$ denotes the mass of consumers accessing at least one content and $\bigcap_{j=0,1} q_{j}$ denotes the number of consumers that access both contents. Given the assumptions taken above, each consumer that access at least a $\mathrm{CP}\left(\bigcup_{j=0,1} q_{j}\right)$ is impressed at least once by each ad. Consumers that access both $\operatorname{CPs}\left(\bigcap_{j=0,1} q_{j}\right)$ are impressed twice. Suppose instead the advertiser single-homes, buying an ad space only from CP $j$. Its total surplus would be

$$
\left(V^{\prime}-p_{j}\right) q_{j} \quad j=0,1
$$

Obviously, the surplus is zero if no ad space is bought. Taking as given the network configuration and prices $p_{j}$, the advertiser will choose the option that guarantees the highest surplus. We may therefore compute total advertisers surplus as

$$
A S=\sum_{j=0,1} d_{s j}\left(V^{\prime}-p_{j}\right) q_{j}+d_{m}\left(V^{\prime} \bigcup_{j=0,1} q_{j}+V^{\prime \prime} \bigcap_{j=0,1} q_{j}-\sum_{j=0,1} p_{j} q_{j}\right)
$$

where $d_{s j}$ is the number of advertisers that single-home on CP $j$ and $d_{m}$ is the number of advertisers that multi-home.

Welfare. We define social welfare $S W$ as the sum of consumer surplus, advertisers surplus and profits of ISPs and CPs. Thus

$$
S W=C S+A S+\sum_{i=A, B} \pi_{i}+\sum_{j=0,1} \pi_{j}
$$

It is intuitive that social welfare coincides with the gross surplus generated by connections at the two ends of the market, i.e. with the sum of gross surplus for consumers and advertisers. Indeed, payments collected by ISPs and CPs are simply transfers from other players and cancel out in $S W$. Replacing $C S, A S, \sum_{i=A, B} \pi_{i}$ and $\sum_{j=0,1} \pi_{j}$ in $S W$, we get

$$
S W=Z+\sum_{i=A, B} \Delta_{i}+\frac{t}{2}-t q_{B}-t q_{A}^{2}+\sum_{j=0,1} d_{s j} V^{\prime} q_{j}+d_{m}\left(V^{\prime} \bigcup_{j=0,1} q_{j}+V^{\prime \prime} \bigcap_{j=0,1} q_{j}\right)
$$

We can conclude the following

Lemma 1 Universal connection and advertisers multi-homing are socially optimal.

PROOF: The fact that each CP is connected to both ISPs implies that $q_{j}=1 \forall j$, as consumers have access to all contents, irrespectively of the ISP they subscribe to. It also implies that $q_{A}=q_{B}=1 / 2$, hence total transport cost $\frac{t}{2}-t q_{B}-t q_{A}^{2}$ is minimized. $S W$ is 
strictly increasing in $\sum_{i=A, B} \Delta_{i}$, which is maximized when $I_{i j}=1 \forall i, j$. It is also optimal to have all advertisers multi-home, since $S W$ increases in the number of ad impressions to consumers.

Timing and definition of equilibrium. The timing of the game is as follows:

1. In the unregulated regime, ISP $A$ and $B$ simultaneously set access fees $F_{i}$. In the net neutrality regime, $F_{i}$ is restricted to zero. Having observed fees $F_{i}$, CPs simultaneously decide which ISP to connect to (if any).

2. ISP $A$ and $B$ simultaneously set subscription fees $a_{i}$. CPs simultaneously set (perimpression) prices $p_{j}$ for their ad spaces. Advertisers buy ad spaces from CPs.

3. Consumers connect to their preferred ISP and visit the available CPs, getting exposed to the ads.

We now provide the definition of an equilibrium in our game. A subgame-perfect equilibrium of the game is a set of fees, prices, connection decisions and demands such that, at each stage, no player wants to deviate given the choice of other players and what has been decided at previous stages. We assume all agents have perfect foresight. The model is solved by backward induction.

A remark is in order at this point. In a standard two-sided market framework, it may occur that ISPs cut subscription fees in order to attract more consumers and exploit network externalities on the content side. The timing we assume rules this out, since price competition for consumers comes at a later stage with respect to competition for contents. Hence, ISPs take revenues collected on the content side as given when deciding on subscription fees. We believe this to be a reasonable simplification, justified by the fact that contracts between ISPs and CPs have generally a more long-run perspective and their connection decision is more rigid than that between ISPs and consumers. ${ }^{21}$

\section{$4 \quad$ ISP monopoly}

In order to understand the forces at work in the model, we start from a scenario in which only one ISP (say, $A$ ) is active. We simply disregard the presence of ISP $B$. To describe in a concise way the network configurations that may arise in equilibrium, we now introduce some notation. In the following, network configuration $(0 A, 1 A)$ means that both 0 and 1 connect to $A$ (i.e. $I_{A j}=1 \forall j$ ): we refer to this configuration (in the monopoly scenario) as

\footnotetext{
${ }^{21}$ A similar assumption is made by Choi et al. (2012) and Kourandi et al. (2013).
} 

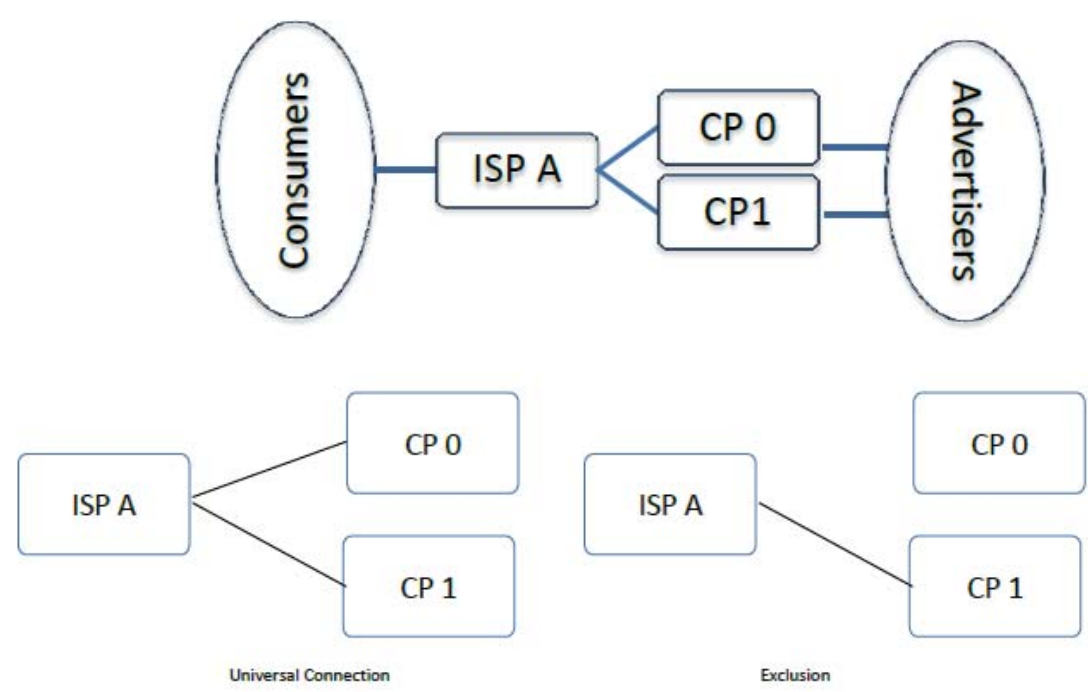

Figure 1: Monopoly setup and possible configurations

"universal connection"; $(0 A, 1 N)$ means that 0 connects to $A$ and 1 connects to no ISP (i.e. $\left.I_{A 0}=1 I_{A 1}=0\right) ;(0 N, 1 N)$ means that no CP connects to any ISP. See Figure 1 below for an illustration.

\subsection{Stage 3}

At Stage 3, consumers decide whether to connect to ISP $A$ or stay out of the market. Indeed, only consumers that are sufficiently close to $A$ (i.e. with low $x$ ) participate in the market. ${ }^{22}$ To compute consumers' demand, we define the marginal consumer $\underline{x}$ who is indifferent between connecting to ISP $A$ and not accessing the Internet at all. From equation $U_{A}(x)=0$, we find

$$
\underline{x}=\frac{Z+\Delta_{A}-a_{A}}{t}
$$

Demand for ISP $A$ is given by all consumers who get positive utility from connecting to the ISP, that is $q_{A}=\underline{x}$.

\section{$4.2 \quad$ Stage 2}

At Stage 2, ISP $A$ decides the subscription fee $a_{A}$ maximizing profits. ISP $A$ 's profits are $\pi_{A}=q_{A} a_{A}+\sum_{j} F_{A} I_{A j}$, where $q_{A}=\underline{x}$. Using the first order condition $\frac{\partial \pi_{A}}{\partial a_{A}}=0$, we find

\footnotetext{
${ }^{22}$ We here relax the assumption that all market is covered.
} 


$$
a_{A m o n}^{*}=\frac{Z+\Delta_{A}}{2}
$$

By substitution, we obtain equilibrium demand and profit: ${ }^{23}$

$$
q_{\text {Amon }}^{*}=\frac{Z+\Delta_{A}}{2 t} \quad \pi_{\text {Amon }}^{*}=\frac{\left(Z+\Delta_{A}\right)^{2}}{4 t}+\sum_{j} F_{A} I_{A j}
$$

Not surprisingly, both subscription fee and demand are increasing in $\Delta_{A}$ and $Z$, and demand is decreasing in $t$. Hence, taking access fee $F_{A}$ as given, profits are increasing in $Z$ and $\Delta_{A}$, and decreasing in $t$.

At this Stage, CPs also set prices for advertising spaces. In order to understand how prices are formed, it is useful to recall how much reaching consumers is worth to advertisers. The expected value of reaching a consumer through an ad on CP $j$ exclusively is $V^{\prime}$. If the advertiser reaches the same consumer also on $\mathrm{CP} j^{\prime}$ exposing the consumer to the ad twice, the additional impression is worth (in expected terms) $V^{\prime \prime}$. If a content provider has exclusive access to consumers attention, it can always charge monopoly prices for ad spaces. On the contrary, if multiple CPs reach the same consumer, competition to attract advertisers brings to lower prices, as in a standard oligopoly model (Ambrus, Calvano and Reisinger, 2011, and Anderson, Foros and Kind, 2011). ${ }^{24}$

To illustrate, suppose to be in the universal distribution configuration. We now argue that the equilibrium is such that CPs charge a per-impression price $p_{j}=V^{\prime \prime} j=0,1$ and advertisers multi-home. To see why, consider that a higher price could profitably be undercut by the rival CP, winning it the entire market. This is because advertisers would optimally single-home on the cheapest outlet. On the other hand, there is no point in charging less than $V^{\prime \prime}$. This is because advertisers would anyway multi-home, since the price per impression is lower than the value of an additional impression $V^{\prime \prime}$. Hence, the price in the competitive case is equal to the value of reaching a consumer through an additional channel. Suppose, instead, CP $j$ were the only one available to subscribers at ISP $A$. It can set a per-impression price $p_{j}=V^{\prime}$, capturing the entire advertiser gross surplus. Obviously, advertisers join only the $\mathrm{CP}$ with a positive market share.

The above discussion introduces us to Lemma 2, presenting CPs' profits at Stage 2. We provide in its proof a more formal and complete description of how ad space prices are determined.

\footnotetext{
${ }^{23}$ Since we have assumed $1 \geq q_{\text {Amon }}^{*}>0$, this implies that we assume $\delta(1+\gamma) \leq 2 t-Z$ holds.

${ }^{24}$ This crucially depends on multi-homing by advertisers and the loss in value of repeated impressions. If multiple impressions did not lose value, competition among CPs would have no impact on prices of ad spaces. If multi-homing of advertisers were not possible, the equilibrium price would be equal to the marginal cost of providing ad spaces, which is zero by assumption.
} 
Lemma 2 In equilibrium, advertisers buy ad slots from all CPs that have access to consumers. Advertising profits $\pi_{j}$ of $C P j$, conditionally on its connection status, are reported in the following table

\begin{tabular}{|c|c|c|}
\hline$\pi_{1}$ & $\mathrm{~A}$ & $\mathrm{No}$ \\
\hline \hline$\pi_{0}$ & $V^{\prime \prime} q_{m o n}^{++}-F_{A}$ & 0 \\
$\mathrm{~A}$ & $V^{\prime \prime} q_{m o n}^{++}-F_{A}$ & $V^{\prime} q_{m o n}^{+}-F_{A}$ \\
& $V^{\prime} q_{m o n}^{+}-F_{A}$ & 0 \\
No & 0 & 0 \\
\hline
\end{tabular}

where $q_{\text {mon }}^{++}=\frac{Z+\delta(1+\gamma)}{2 t}$ and $q_{\text {mon }}^{+}=\frac{Z+\delta}{2 t}$

Table 1: Monopoly model: advertising profits $\pi_{j}$ of $\mathrm{CP} j$, conditionally on its connection status.

PROOF: We consider each possible market configuration in turn. Consider first the universal distribution case. Thus, $q_{j}=q_{A}=q_{\text {mon }}^{++} j=0,1$. Suppose an advertiser puts ads on both CPs. Its total surplus would be $\left(V^{\prime}+V^{\prime \prime}-\sum_{j=0,1} p_{j}\right) q_{m o n}^{++}$. Suppose instead the advertiser decides to put ads only on CP $j$. Its total surplus is $\left(V^{\prime}-p_{j}\right) q_{m o n}^{++}$since, in this case, consumers are exposed only to first impressions. This implies that an advertiser prefers to put ads on both CPs rather than on one if and only if $V^{\prime \prime} \geq p_{j}$. We now prove that $p_{j}=V^{\prime \prime} j=0,1$ is the equilibrium price schedule for advertising spaces and that advertisers multi-home. Suppose $p_{j^{\prime}} \leq V^{\prime \prime}$ : as long as $p_{j} \leq V^{\prime \prime}$ advertisers would optimally multi-home and put ads on both CPs, while if $p_{j}>V^{\prime \prime}$ no platform would join CP $j$. Hence, it is optimal for CP $j$ to set $p_{j}=V^{\prime \prime}$. Suppose instead that $p_{j^{\prime}}>V^{\prime \prime}$ : then, it is optimal for $j$ to set $p_{j}=p_{j^{\prime}}-\varepsilon$. By so doing, all advertisers would single-home and buy only ad slots from $j$, leaving the other $\mathrm{CP}$ with zero revenues. It follows that the only equilibrium prices (mutual best response) are such that $p_{j}=V^{\prime \prime} \quad j=0,1$. It is easily seen that, in such equilibrium, all advertisers multi-home. Finally, each CP makes a profit equal to $V^{\prime \prime} q_{\text {mon }}^{++}-F_{A}$.

Suppose now the market configuration involved exclusion, i.e. either $(0 \mathrm{~A}, 1 \mathrm{~N})$ or $(0 \mathrm{~N}, 1 \mathrm{~A})$. For instance, consider configuration $(0 \mathrm{~A}, 1 \mathrm{~N})$. If an advertiser puts ads on both $\mathrm{CPs}$, its total surplus is $\left(V^{\prime}-\sum_{j=0,1} p_{j}\right) q_{m o n}^{+}$. If ads are put only on CP 0 , the surplus is $\left(V^{\prime}-p_{0}\right) q_{m o n}^{+}$. 
If instead ads are put only on CP 1 , the surplus is $0-p_{1}$. We now prove that CPs set the following equilibrium prices: $p_{1}=0$ and $p_{0}=V^{\prime}$. Indeed, as CP 1 does not provide any surplus to advertisers (since it is not able to reach consumers), they never join its platform for any positive price. Instead, CP 0 has no incentive to fix any price lower than $V^{\prime}$, since advertisers join this platform for a price up to this value. Hence, CP 1's profits are $\pi_{1}=0$ and CP 0's profits are $\pi_{0}=V^{\prime} q_{m o n}^{+}-F_{A}$.

Trivially, when no CP is connected, no advertising takes place. Hence, all profits are zero.

\subsection{Stage 1}

At stage 1, ISP $A$ sets the access fee $F_{A}$ and CPs decide whether to connect. This determines the network configuration. We now present the subgame-perfect equilibria of the game. We begin from the benchmark case of net neutrality, where the access fee is constrained to zero. Next, we consider the unregulated scenario, in which ISP $A$ freely sets $F_{A}$. In the following, we denote with superscript $N$ the equilibrium variables under net neutrality, and with superscript $U$ those under the unregulated regime.

\subsubsection{Benchmark: net neutrality regime}

In the network neutrality case, ISP profits are simply given by revenues collected from subscription fees on the consumers' market

$$
\pi_{\text {Amon }}^{N}=\frac{\left(Z+\Delta_{A}\right)^{2}}{4 t} \quad \text { since } \quad F_{A}^{N}=0
$$

We now study CPs' connection decisions, and derive the corresponding configuration of the network. Elaboration from the table provided in Lemma 2 leads to the following

Proposition 1 Suppose there is a single ISP. In the net neutrality regime there exists a unique subgame-perfect equilibrium entailing universal distribution (i.e. where the market configuration is $(0 A, 1 A))$. It is such that

$$
a_{\text {Amon }}^{N}=\frac{Z+\delta(1+\gamma)}{2} \quad q_{A}^{N}=q_{j}^{N}=\frac{Z+\delta(1+\gamma)}{2 t} \quad p_{j}^{N}=V^{\prime \prime} \quad I_{A j}^{N}=1 \forall j
$$

PROOF: Immediate from Lemma 2, when $F_{i}=0$.

Under net neutrality, universal connection is the unique equilibrium. Observe, however, that when both CPs are connected to the monopolist ISP, they cannot charge the monopoly 
price $V^{\prime}$ for ad slots. Each CP would be better off if the rival were excluded from the market. Yet, since connection is free, this never takes place in equilibrium.

\subsubsection{Unregulated regime}

In the unregulated regime, ISP $A$ is allowed to impose an access fee $F_{A}$ to CPs. The following Proposition presents the subgame-perfect equilibria in this regime:

Proposition 2 Suppose there is a single ISP. In the unregulated regime:

- if and only if the value of second ad impressions is high enough, i.e.

$$
V^{\prime \prime} \geq\left(V^{\prime} \frac{Z+\delta}{2}-\frac{\delta \gamma(2 Z+2 \delta+\delta \gamma)}{4}\right) \frac{1}{Z+\delta(1+\gamma)}
$$

there exists a subgame-perfect equilibrium entailing universal distribution (i.e. market configuration $(0 A, 1 A))$. It is such that

$$
F_{A}^{U}=V^{\prime \prime} q_{m o n}^{++} \quad a_{A}^{U}=\frac{Z+\delta(1+\gamma)}{2} \quad q_{A}^{U}=q_{j}^{U}=q_{m o n}^{++}=\frac{Z+\delta(1+\gamma)}{2 t} \quad p_{j}^{U}=V^{\prime \prime} \quad I_{A j}^{U}=1 \forall j
$$

- if and only if the value of second ad impressions is low enough, i.e. if

$$
0<V^{\prime \prime}<\left(V^{\prime} \frac{Z+\delta}{2}-\frac{\delta \gamma(2 Z+2 \delta+\delta \gamma)}{4}\right) \frac{1}{Z+\delta(1+\gamma)}
$$

there exists a subgame-perfect equilibrium entailing exclusion of one CP (i.e. market configuration $(0 A, 1 N)$ or $(O N, 1 A))$. It is such that

$$
F_{A}^{U}=V^{\prime} q_{m o n}^{+} \quad a_{i}^{U}=\frac{Z+\delta}{2} \quad q_{A}^{U}=q_{j}^{U}=q_{m o n}^{+}=\frac{Z+\delta}{2 t} \quad p_{j}^{U}=V^{\prime} \quad \sum_{j=0,1} I_{A j}^{U}=1 \forall j
$$

PROOF: see the Appendix.

Differently from the net neutrality regime, the ISP can influence the network configuration by setting the access fee. Depending on parameter values, the equilibrium is either such that both content providers connect to the ISP or that only one does. Since competition reduces the profits a $\mathrm{CP}$ can obtain on the advertising market, each is ready to pay a premium for being the only outlet to reach consumers. By setting a high access fee (equal to $V^{\prime}$ per consumer reached), the ISP discourages one CP from connecting to its network. At the same time, it extracts the high profits made by the $\mathrm{CP}$ that does connect. On the other hand, since 


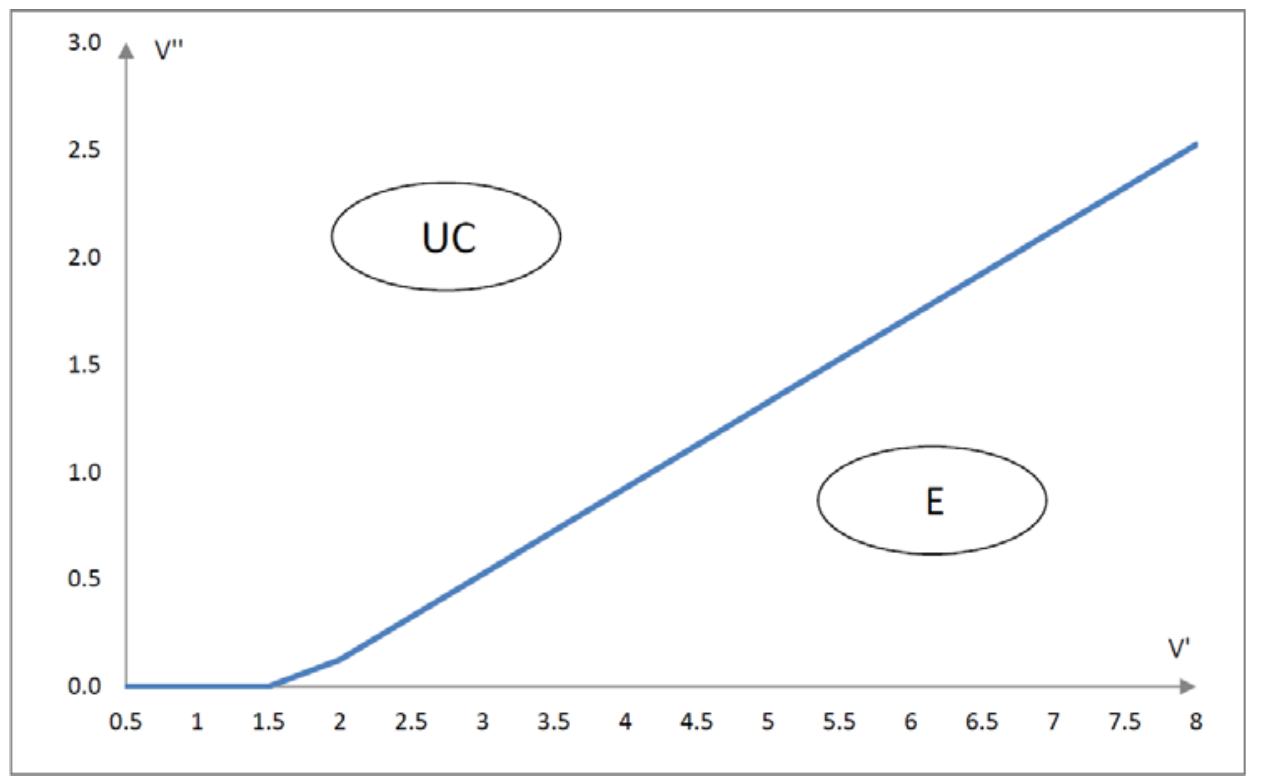

Figure 2: Monopoly, equilibria in the unregulated regime: the blue line represents threshold $\left(V^{\prime} \frac{Z+\delta}{2}-\frac{\delta \gamma(2 Z+2 \delta+\delta \gamma)}{4}\right) \frac{1}{Z+\delta(1+\gamma)}$. UC stands for Universal Connection and E for Exclusion. Graphs are obtained setting $\delta=0.5$ and $\gamma=1$.

the attractiveness of its platform is reduced, the high access fee also implies the ISP renounces to a share of consumers (who subscribe only if multiple contents are available). Alternatively, the ISP can choose a lower fee (i.e. equal to the value of a second impression $V^{\prime \prime}$ per each consumer), inducing universal connection. Hence, the surplus per consumer extracted from each CP is lower, but the consumer base is maximized. Moreover, the access fee is collected from both CPs.

We see therefore that the equilibrium crucially depends on the value of first and repeated ad impressions, $V^{\prime}$ and $V^{\prime \prime}$, as well as on the attractiveness of content for consumers. If ads lose little value when repeated (i.e. $V^{\prime \prime}$ is close enough to $V^{\prime}$ ), CPs have a substantial willingness to pay for connection even if they have to compete for consumers' attention. Hence, the ISP prefers to induce universal connection. Otherwise, the ISP induces total fragmentation. Figure 2 shows the equilibria conditionally on the values of $V^{\prime \prime}$ and $V^{\prime}$.

Finally, we see that the value of contents for consumers and their complementarity induce the ISP to attract more CPs. This is easily explained: if contents are valuable and not highly substitutable (i.e. both $\delta$ and $\gamma$ are high enough), the loss of consumer base when excluding a content is significant. It follows that the ISP is better off attracting both content providers. If instead $\delta$ and $\gamma$ are low enough, excluding a CP has a small cost in terms of foregone consumer subscriptions. 


\subsection{Comparison of the two regulatory regimes}

Let us now briefly compare the two regulatory regimes with a monopolist ISP. While CPs are always universally distributed under net neutrality, one of them might be excluded from the network in the unregulated regime. However, the results also indicate that, by itself, the removal of net neutrality is not sufficient to produce exclusion of content. Exclusion crucially depends on how much consumers value content availability and on how competition among CPs affects profitability of advertising spaces.

The above has very intuitive welfare implications. Consumers are always better off with universal connection than with exclusion, since they have access to more contents. Advertisers are also better off under universal distribution, since the number of ad impressions increases and their price goes down. Consequently, consumers and advertisers are strictly better off with net neutrality, as long as advertising spaces strongly lose profitability in the presence of competition. Otherwise, consumers and advertisers are indifferent between the two regulatory regimes, since universal connection will emerge regardless. On the other hand, CPs are strictly better off under net neutrality, since under the unregulated regime all their profits are extracted by the ISP. For the same reason, the ISP is better off under the unregulated regime. Finally, since social welfare depends essentially on the gross surplus at both ends of the market, we conclude that society as a whole is weakly better off under net neutrality.

\section{ISP duopoly}

Let us now move to the fully-fledged version of the model, where both ISPs are active on the market. Following the notation introduced in Section 4, network configuration $(0 A B, 1 A B)$ will here mean that 0 connects to both $A$ and $B$ and 1 connects to both $A$ and $B$ (i.e. $\left.I_{i j}=1 \forall i, j\right)$; we refer to this configuration, where all CPs are connected to all ISPs, as "universal connection". $(0 A, 1 B)$ means that 0 connects only to $A$ and so does 1 (i.e. $I_{A 0}=$ $\left.I_{B 1}=1 I_{A 1}=I_{B 0}=0\right)$ : we refer to this configuration, where each ISP provides exclusive access to a single (different) $\mathrm{CP}$, as "total fragmentation". $(0 A, 1 A B)$ means that 0 connects to ISP A and 1 connects to both: we refer to this configuration where a CP joins only one ISP, while the other CP joins both ISPs as "partial fragmentation". Finally, $(0 N, 1 A B)$ means that 0 connects to no ISP and 1 connects to both $A$ and $B,\left(I_{A 1}=I_{B 1}=1 I_{A 0}=I_{B 0}=0\right)$. Similar notation is used for all other configurations. See Figure 3 for an illustration. 

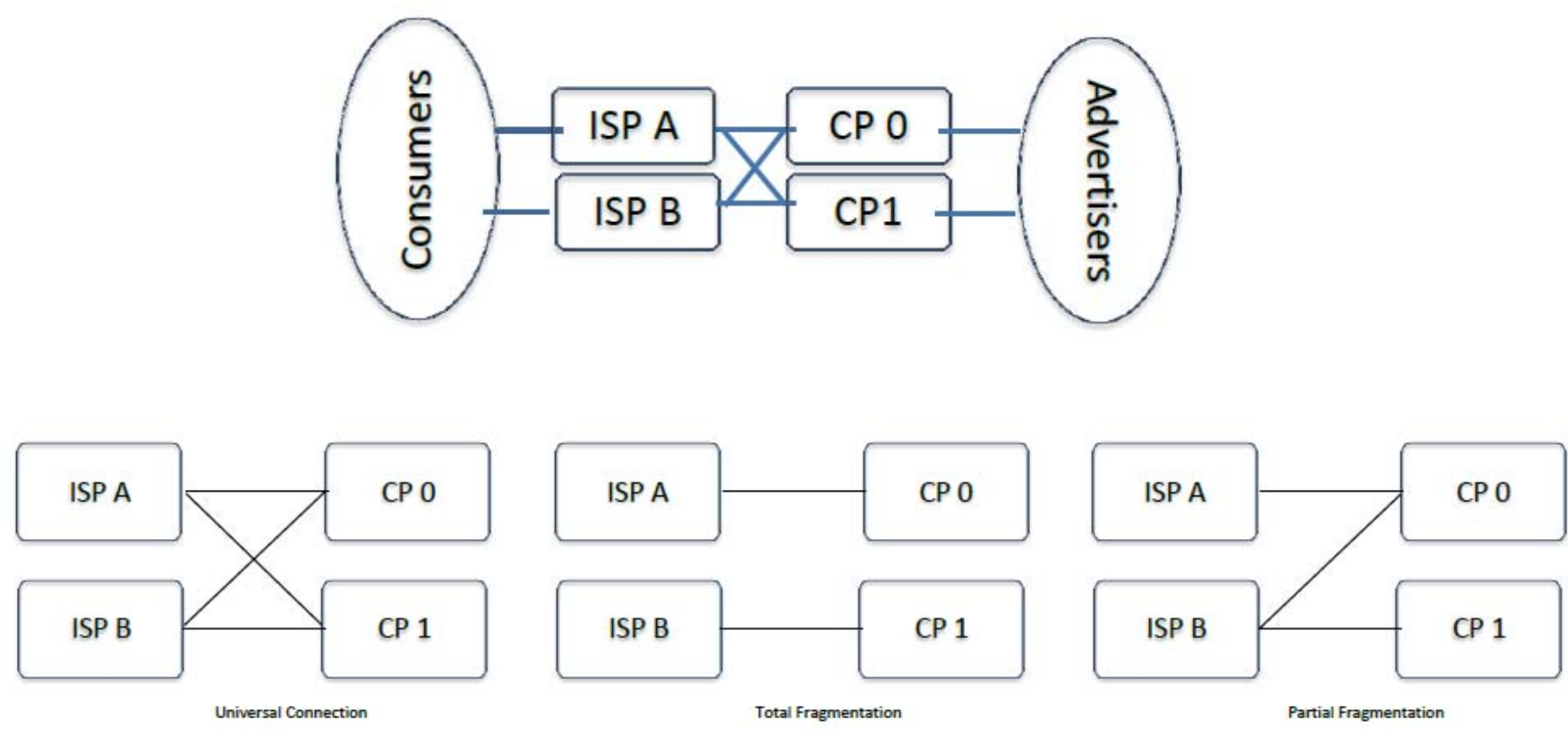

Figure 3: Duopoly setup and network configurations

\section{$5.1 \quad$ Stage 3}

At Stage 3, consumers choose which ISP they connect to, maximizing their utility and taking as given the CPs available at each ISP $i$ and subscription fees $a_{i}$. In order to compute demands, we determine the marginal consumer $\bar{x}$ who is indifferent between the two ISPs. Equalizing $U_{A}(x)=U_{B}(x)$ and solving for $x$ we find

$$
\bar{x}=\frac{1}{2}+\frac{\left(\Delta_{A}-\Delta_{B}\right)-\left(a_{A}-a_{B}\right)}{2 t}
$$

The demand for ISP $A$ is given by all consumers to the left of $\bar{x}$ on the Hotelling line, while that for ISP $B$ is given by all consumers to its right. That is, $q_{A}=\bar{x}$ and $q_{B}=1-\bar{x}$.

\section{$5.2 \quad$ Stage 2}

At Stage 2, ISPs simultaneously set subscription fees, maximizing their profits. They do so taking as given access fees $F_{i}$ as well as CPs' connection decisions (set at Stage 1), and anticipating consumers' behavior at Stage 3. Equilibrium fees are obtained as the solution to the system of first order conditions $\frac{\partial \pi_{i}}{\partial a_{i}}=0 i=A, B$. The result, for any network 
configuration, is

$$
a_{i}^{*}=t+\frac{\left(\Delta_{i}-\Delta_{i^{\prime}}\right)}{3} \quad i=A, B \quad i^{\prime} \neq i
$$

Replacing $a_{i}^{*}$ in $q_{i}$ and $\pi_{i}$, we get demands and profits ${ }^{25}$

$$
q_{i}^{*}=\frac{1}{2}+\frac{\left(\Delta_{i}-\Delta_{i^{\prime}}\right)}{6 t} \quad \pi_{i}^{*}=\frac{\left(3 t+\Delta_{i}-\Delta_{i^{\prime}}\right)^{2}}{18 t}+\sum_{j=0,1} I_{i j} F_{i} \quad i=A, B \quad i^{\prime} \neq i
$$

Profits of platform $i$ are increasing in $\left(\Delta_{i}-\Delta_{i^{\prime}}\right)$. Thus, a uniform increase in quality of both platforms leaves profits unchanged.

In order to describe in a brief way the equilibrium demands conditional on the values of $\Delta_{i}-\Delta_{i^{\prime}}$, we introduce some additional notation, summarized in Table 2 below:

\begin{tabular}{|c|c|c|c|c|c|}
\hline$\Delta_{i}-\Delta_{i^{\prime}}$ & $q_{i}^{*}$ & defined as & $\Delta_{i}-\Delta_{i^{\prime}}$ & $q_{i}^{*}$ & defined as \\
\hline$-\delta_{1}$ & $\frac{1}{2}-\frac{\delta}{6 t}$ & $q_{i}^{--}$ & $\delta_{1}$ & $\frac{1}{2}+\frac{\delta}{6 t}$ & $q_{i}^{++}$ \\
\hline$-\delta_{2}$ & $\frac{1}{2}-\frac{\delta \gamma}{6 t}$ & $q_{i}^{-}$ & $\delta_{2}$ & $\frac{1}{2}+\frac{\delta \gamma}{6 t}$ & $q_{i}^{+}$ \\
\hline 0 & $\frac{1}{2}$ & & 0 & $\frac{1}{2}$ & \\
\hline$-\left(\delta_{1}+\delta_{2}\right)$ & $\frac{1}{2}-\frac{\delta(1+\gamma)}{6 t}$ & $q_{i}^{---}$ & $\delta_{1}+\delta_{2}$ & $\frac{1}{2}+\frac{\delta(1+\gamma)}{6 t}$ & $q_{i}^{+++}$ \\
\hline
\end{tabular}

Table 2: Notation for demand of ISP $i$, duopoly case.

CPs set prices for ad spaces in the same way as in the monopoly case of Section 4. However, we can now find situations where a CP is the unique gatekeeper only for a part of consumers connected to the Internet (the remaining part being reached also by the other $\mathrm{CP}$ ). In this case, as we formally show in Lemma 3, prices for ad spaces are a weighted average of the value of first impression $V^{\prime}$ and second impression $V^{\prime \prime}$, the weights being respectively the demand of subscribers who visit only one CP and the demand of subscribers who visit both CPs. Lemma 3 presents CP profits at Stage 2.

Lemma 3 All advertisers multi-home at equilibrium. Profits $\pi_{j}$ of $C P j=0,1$, conditional on the network configuration, are as reported in the following table

\footnotetext{
${ }^{25}$ Since we have assumed $\sum q_{i}^{*}=1$ and $q_{i}^{*}>0 \forall i$, this implies that we assume $\delta(1+\gamma)<3 t$ holds.
} 


\begin{tabular}{|c|c|c|c|c|}
\hline$\pi_{1}$ & $\mathrm{~A} \& \mathrm{~B}$ & $\mathrm{~A}$ & $\mathrm{~B}$ & None \\
\hline \hline \multirow{3}{*}{$\mathrm{A} \& \mathrm{~B}$} & $V^{\prime \prime}-\sum F_{i}$ & $V^{\prime \prime} q^{+}-F_{A}$ & $V^{\prime \prime} q^{+}-F_{B}$ & 0 \\
& $V^{\prime \prime}-\sum F_{i}$ & $V^{\prime \prime} q^{+}+V^{\prime} q^{-}-\sum F_{i}$ & $V^{\prime \prime} q^{+}+V^{\prime} q^{-}-\sum F_{i}$ & $V^{\prime}-\sum F_{i}$ \\
\hline & $V^{\prime \prime} q^{+}+V^{\prime} q^{-}-\sum F_{i}$ & $V^{\prime \prime} q^{+++}-F_{A}$ & $\frac{V^{\prime}}{2}-F_{B}$ & 0 \\
$\mathrm{~A}$ & $V^{\prime \prime} q^{+}-F_{A}$ & $V^{\prime \prime} q^{+++}-F_{A}$ & $\frac{V^{\prime}}{2}-F_{A}$ & $V^{\prime} q^{++}-F_{A}$ \\
\hline \multirow{2}{*}{$\mathrm{B}$} & $V^{\prime \prime} q^{+}+V^{\prime} q^{-}-\sum F_{i}$ & $\frac{V^{\prime}}{2}-F_{A}$ & $V^{\prime \prime} q^{+++}-F_{B}$ & 0 \\
& $V^{\prime \prime} q^{+}-F_{A}$ & $\frac{V^{\prime}}{2}-F_{B}$ & $V^{\prime \prime} q^{+++}-F_{B}$ & $V^{\prime} q^{++}-F_{B}$ \\
\hline \multirow{2}{*}{ None } & $V^{\prime}-\sum F_{i}$ & $V^{\prime} q^{++}-F_{A}$ & $V^{\prime} q^{++}-F_{B}$ & 0 \\
& 0 & 0 & 0 & 0 \\
\hline
\end{tabular}

Table 3: Duopoly model: advertising profits $\pi_{j}$ of $\mathrm{CP} j$, conditionally on connection status.

PROOF: We consider each of the possible market configurations in turn. First, consider universal distribution. If an advertiser puts ads on both CPs, its total surplus would be $V^{\prime}+V^{\prime \prime}-p_{0}-p_{1}$. If instead ads are put only on $\mathrm{CP} i$, the surplus is $V^{\prime}-p_{i}$. The discussion concerning this configuration is the same as the one in the proof of Lemma 2 for the configuration $(0 \mathrm{~A}, 1 \mathrm{~A})$.

Suppose now the market configuration involves partial fragmentation. For instance, consider the case $(0 \mathrm{AB}, 1 \mathrm{~A})$. If an advertiser puts ads on both $\mathrm{CPs}$, its total surplus would be $\left(V^{\prime}-p_{0}\right) q^{-}+\left(V^{\prime}+V^{\prime \prime}-p_{0}-p_{1}\right) q^{+}$. If instead ads are put only on CP 0 , the surplus is $V^{\prime}-p_{0}$. Finally, if ads are put only on CP 1 , its surplus is $\left(V^{\prime}-p_{1}\right) q^{+}$. We now prove that ISPs set the following equilibrium prices: $p_{1}=V^{\prime \prime}$ and $p_{0}=V^{\prime} q^{-}+V^{\prime \prime} q^{+}$and that advertisers multi-home. Let us start by describing the best response function for CP 0. Suppose $p_{1} \leq V^{\prime \prime}$. In that case, advertisers would buy ad spaces from CP 1 , irrespectively of whether they have already bought ads from CP 0 or not, since price is surely below the value of a repeated impression. If they put ads on $\mathrm{CP} 1$, however, they will also put them on $\mathrm{CP} 0$ only if $p_{0}$ is low enough, i.e. only if

$$
\left(V^{\prime}-p_{0}\right) q^{-}+\left(V^{\prime}+V^{\prime \prime}-p_{0}-p_{1}\right) q^{+} \geq\left(V^{\prime}-p_{1}\right) q^{+}
$$


which results in $p_{0} \leq V^{\prime} q^{-}+V^{\prime \prime} q^{+}$. It follows that the best response for CP 0 is to set $p_{0}=V^{\prime} q^{-}+V^{\prime \prime} q^{+}$(otherwise, CP 0 would not be able to sell any ad space). Suppose now $p_{1}>V^{\prime \prime}$ : in that case, advertisers putting ads on CP 0 , would never put them on $\mathrm{CP} 1$, the price being too high. This rules out multi-homing, so all advertisers put ads either on CP 0 or CP 1. Clearly, CP 0 is better off being the player capturing all the market. This happens if and only if

$$
\left(V^{\prime}-p_{0}\right) \geq\left(V^{\prime}-p_{1}\right) q^{+}
$$

holds. As a consequence, the best response is $p_{0}=V^{\prime} q^{-}+p_{1} q^{+}$.

Let us now describe the best response function for CP 1. Suppose first that $p_{0} \leq V^{\prime} q^{-}+$ $V^{\prime \prime} q^{+}$. Advertisers multi-home if and only if $p_{1} \leq V^{\prime \prime}$. Otherwise, they all converge on one of the two CPs. However, if $p_{1}>V^{\prime \prime}$, this CP is 0 , as condition

$$
\left(V^{\prime}-p_{0}\right) \geq\left(V^{\prime}-p_{1}\right) q^{+}
$$

is holds. It follows that the best response is $p_{1}=V^{\prime \prime}$. Suppose now that $p_{0}>V^{\prime} q^{-}+V^{\prime \prime} q^{+}$. $p_{0}$ is sufficiently high for CP 1 to be able to capture all the market while still setting a higher price than $V^{\prime \prime}$. Indeed, it can easily be verified that condition

$$
\left(V^{\prime}-p_{0}\right) \leq\left(V^{\prime}-p_{1}\right) q^{+}
$$

holds as long as $p_{1} \leq \frac{p_{0}-V^{\prime} q^{-}}{q^{+}}$. The threshold on the right hand side of the inequality is strictly higher than $V^{\prime \prime}$ as long as $p_{0}>V^{\prime} q^{-}+V^{\prime \prime} q^{+}$. Hence, by setting $p_{1}=\frac{p_{0}-V^{\prime} q^{-}}{q^{+}}>V^{\prime \prime}$, CP 1 is able to capture all the market. Hence, the best response is $p_{1}=\frac{p_{0}-V^{\prime} q^{-}}{q^{+}}$.

We are now in a position to describe the equilibrium. It is quite easy to see that the only mutual best response is such that $p_{1}=V^{\prime \prime}$ and $p_{0}=V^{\prime} q^{-}+V^{\prime \prime} q^{+}$. This implies that CPs' profits are $\pi_{1}=V^{\prime \prime} q^{+}-F_{A}$ and $\pi_{0}=V^{\prime} q^{-}+V^{\prime \prime} q^{+}-\sum F_{i}$ and that advertisers multi-home. A similar reasoning applies when the configuration is $(0 \mathrm{~A}, 1 \mathrm{AB}),(0 \mathrm{~B}, 1 \mathrm{AB})$ and $(0 \mathrm{AB}, 1 \mathrm{~B})$.

Consider now a configuration with all CPs being distributed by only one ISP, i.e. either $(0 \mathrm{~A}, 1 \mathrm{~A})$ or $(0 \mathrm{~B}, 1 \mathrm{~B})$. With a reasoning similar to that of the case of universal connection, one can prove that the only equilibrium is such that $p_{0}=p_{1}=V^{\prime \prime}$ so that CPs' profits are $\pi_{1}=\pi_{0}=V^{\prime \prime} q^{++}-F_{A}$. Again, advertisers multi-home.

Consider a market configuration involving full segmentation, i.e. either $(0 \mathrm{~A}, 1 \mathrm{~B})$ or $(0 \mathrm{~B}, 1 \mathrm{~A})$. In this case, each $\mathrm{CP}$ would be a monopolistic outlet for the consumers it reaches. It is quite intuitive (we omit the proof) that equilibrium prices are $p_{0}=p_{1}=V^{\prime}$ and profits $\pi_{1}=\pi_{0}=\frac{V^{\prime}}{2}-F_{i}$. It is again the case that, in equilibrium, advertisers multi-home. 
Consider now a configuration with one CP being distributed by both ISPs, while the other CP being distributed by no ISP. Take, for example, the case $(0 \mathrm{AB}, 1 \mathrm{~N})$. No advertiser puts an ad on CP 1 since no consumer can reach it. If an advertiser puts ads on CP 0, its total surplus is $V^{\prime}-p_{0}$. Thus, quite simply, the only equilibrium is such that $p_{0}=V^{\prime}$. Hence, CPs' profits are $\pi_{1}=0, \pi_{0}=V^{\prime}-\sum F_{i}$. In this case, advertisers single-home, since they have no benefit from advertising on $\mathrm{CP} 1$. A similar reasoning applies to configuration $(0 \mathrm{~N}, 1 \mathrm{AB})$.

Finally, consider a configuration with one CP being distributed by one ISP, with the other $\mathrm{CP}$ being distributed by none, such as $(0 \mathrm{~A}, 1 \mathrm{~N})$. No advertiser puts an ad on CP1 since no consumer is reached. If an advertiser puts ads on CP 0 , its total surplus is $\left(V^{\prime}-p_{0}\right) q^{+}$. Thus, $p_{0}=V^{\prime}$. Hence, CPs' profits are $\pi_{1}=0, \pi_{0}=V^{\prime} q^{+}-F_{A}$. A similar reasoning applies when the configuration is $(0 \mathrm{~N}, 1 \mathrm{~A}),(0 \mathrm{~N}, 1 \mathrm{~B})$ and $(0 \mathrm{~B}, 1 \mathrm{~N})$.

\section{$5.3 \quad$ Stage 1}

At Stage 1, ISP $i$ sets the access fee $F_{i}$ and CPs decide to which ISP they want to connect, if any. Our objective is now to determine the subgame-perfect equilibria of the game. We begin from the benchmark case of net neutrality, where access fees $F_{i}$ are constrained to zero. Next, we consider the unregulated scenario, in which ISPs freely set $F_{i}$.

\subsubsection{Benchmark: net neutrality regime}

In the net neutrality case, profits for ISPs are simply given by revenues collected from subscription fees

$$
\pi_{i}=\frac{\left(3 t+\Delta_{i}-\Delta_{-i}\right)^{2}}{18 t} \quad i=A, B
$$

We now check which configuration of the network occurs at equilibrium by studying CPs' connection choices, given $F_{i}=0$. Elaborating from Lemma 3, we have

Proposition 3 In the net neutrality regime:

- there always exists a subgame-perfect equilibrium entailing universal connection (i.e. market configuration $(0 A B, 1 A B))$. It is such that

$$
a_{i}^{N}=t \quad q_{i}^{N}=\frac{1}{2} \quad p_{j}^{N}=V^{\prime \prime} \quad q_{j}^{N}=1 \quad I_{i j}^{N}=1 \forall i, j
$$

- if and only if the value of second impressions of advertisements is low enough, i.e. $V^{\prime \prime}<$ $V^{\prime}\left(\frac{\delta \gamma}{3 t+\delta \gamma}\right)$, there also exists a subgame-perfect equilibrium entailing total fragmentation 
(i.e. market configuration $(0 A, 1 B)$ or $(0 B, 1 A))$. It is such that

$$
a_{i}^{N}=t \quad q_{i}^{N}=\frac{1}{2} \quad p_{j}^{N}=V^{\prime} \quad q_{j}^{N}=\frac{1}{2} \sum_{j=0,1} I_{i j}^{N}=1 \quad I_{i j}^{N} \neq I_{-i j}^{N} \forall i, j
$$

PROOF: Simply follows from the table in Lemma 3, setting $F_{i}=0$.

Under net neutrality, universal connection is always an equilibrium, even though it is not necessarily the most desirable outcome for CPs. When connecting to the same ISP, each $\mathrm{CP}$ produces a strategic externality on its rival, eliminating the possibility to charge the full price $V^{\prime}$ for ad slots. It follows that, when the price of ad spaces is strongly reduced by competition (i.e. $V^{\prime \prime}$ is significantly smaller than $V^{\prime}$ ) CPs could make higher profits if each joined a different ISP, fragmenting the network. Yet, since connection is free, the externality is uninternalized and, as a result, universal connection obtains. In other words, CPs find themselves in a prisoner's dilemma, to the benefit of consumers and advertisers.

In contrast to the monopoly case studied in the previous Section, net neutrality is not sufficient to guarantee universal connection. There also exists the possibility of a second equilibrium, characterized by total fragmentation. This occurs when competition among CPs strongly affects their profitability (i.e. $V^{\prime \prime}$ is small compared to $V^{\prime}$ ) and/or contents are highly valuable to consumers and complementary. Indeed, the threshold $V^{\prime}\left(\frac{\delta \gamma}{3 t+\delta \gamma}\right)$ is strictly increasing in $\delta$ and $\gamma$. Hence, if ad impressions rapidly lose value when repeated and content is important for attracting consumers, fragmentation can occur even with net neutrality regulation.

The last finding is quite counterintuitive: one would, a priori, expect that the more consumers care about content and the greater their complementarity, the more CPs would try to take advantage of these forces by connecting to all available ISPs. To see why it is not the case, suppose $(0 \mathrm{~A}, 1 \mathrm{~B})$ to be the market configuration. Consider the problem of a content provider, say 0 , deciding whether to connect also to ISP $B$ or not. If it does not connect to $B$, the price it can charge for ad spaces is $V^{\prime}$, given that it is the only outlet for advertisers wanting to reach $A$ 's consumers. If CP 0 connects to $B$ as well, its consumer base is enlarged, now including also $B$ 's customers. Nevertheless, the price it can charge for ad spaces is now the weighted average $V^{\prime} q^{-}+V^{\prime \prime} q^{+}$. This is because part of its audience would already be reached by the rival content provider. Moreover, by connecting to $B$, CP 0 increases $B$ 's attractiveness for consumers relative to $A$. Hence, though connection to $B$ is free, it has an implicit cost: the quantity of eyeballs CP 0 monopolizes shrinks, since it induces part of $A$ 's consumers to migrate to $B$. This is particularly relevant when contents are valuable for consumers and highly complementary. Thus, if the effect of content availability on consumer 


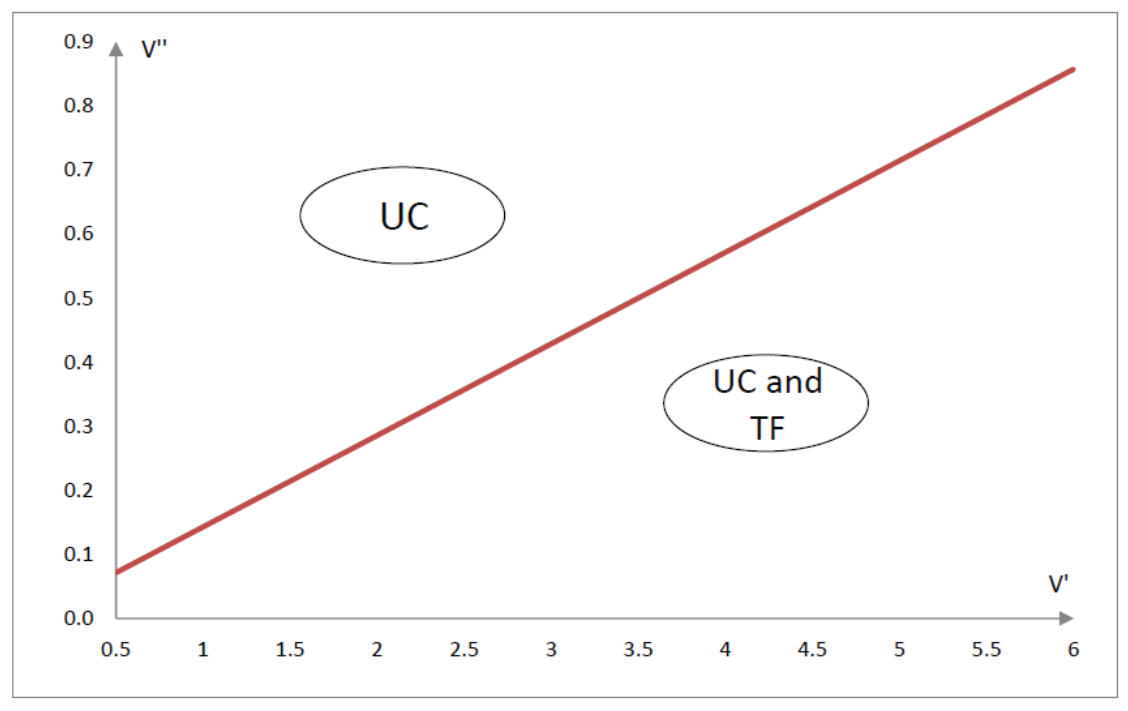

Figure 4: Duopoly, equilibria with Net Neutrality: the red line represents threshold $V^{\prime}\left(\frac{3 t}{6 t+\delta}\right)$. UC stands for Universal Connection and TF for Total Fragmentation. Graphs are obtained setting $\delta=0.5$ and $\gamma=1$.

demand is high enough and/or the value of repeated ad impressions is small, the implicit cost of an additional connection for CP 0 is too high. It follows that it may prefer not to connect to the additional ISP, even if it can do so for free. ${ }^{26}$

\subsubsection{Unregulated regime}

We now study the unregulated regime, where the access fee $F_{i}$ can be freely set. For a given network configuration to arise, the couple $\left(F_{A}, F_{B}\right)$ has to be such that profits of each CP (see Table 3) in the given configuration (and given the choice of the rival) have to be higher than in any other configuration. The equilibria are described in Proposition 4.

Proposition 4 In the unregulated regime:

\footnotetext{
${ }^{26}$ We find that the equilibrium with total fragmentation payoff-dominates the one with universal connection, since $V^{\prime \prime} \leq \frac{V^{\prime}}{2}$ when $V^{\prime \prime}<V^{\prime}\left(\frac{\delta \gamma}{3 t+\delta \gamma}\right)$. On the other hand, the latter risk-dominates the former. Let $r \in$ $(0,1)$ be the probability that $\mathrm{CP} j$ chooses to join both ISPs and $(1-r)$ the probability that it chooses to join either $A$ or $B$. The expected payoff of CP $j$ from joining both ISPs is always higher than the expected payoff from joining only one ISP in the region where both equilibria exist, i.e. $r\left(V^{\prime \prime}\right)+(1-r)\left(V^{\prime} q^{-}+V^{\prime \prime} q^{+}\right)>$ $r\left(V^{\prime \prime} q^{+}\right)+\frac{(1-r)}{2}\left(V^{\prime \prime} q^{+++}\right)+\frac{(1-r)}{2}\left(\frac{V^{\prime}}{2}\right)$ for all possible $r$ when $V^{\prime \prime}<V^{\prime}\left(\frac{\delta \gamma}{3 t+\delta \gamma}\right)$. Hence, the payoff dominance criterion selects the equilibrium that induces total fragmentation, while the risk dominance one selects the equilibrium inducing universal distribution. Harsanyi (1995) established that, when these two criteria lead to different solutions, risk dominant equilibrium should be the chosen one.
} 
- if and only if the value of second ad impressions is high enough, i.e.

$$
V^{\prime}\left(\frac{3 t}{6 t+\delta}\right) \leq V^{\prime \prime} \leq V^{\prime}
$$

there exists a subgame-perfect equilibrium entailing universal distribution (i.e. market configuration $(0 A B, 1 A B))$. It is such that:

$$
F_{i}^{U}=V^{\prime \prime} q^{-} \quad a_{i}^{U}=t \quad q_{i}^{U}=\frac{1}{2} \quad p_{j}^{U}=V^{\prime \prime} \quad q_{j}^{U}=1 \quad I_{i j}^{U}=1 \forall i, j
$$

- if and only if the value of second ad impressions is low enough, i.e. if

$$
0 \leq V^{\prime \prime}<\left(\frac{V^{\prime}}{2}+\frac{t}{2}-\frac{(3 t+\delta(1+\gamma))^{2}}{18 t}\right)\left(\frac{3 t}{3 t+\delta(1+\gamma)}\right)
$$

there exists a subgame-perfect equilibrium entailing total fragmentation (i.e. market configuration $(0 A, 1 B)$ or $(0 B, 1 A))$. It is such that:

$$
F_{i}^{U}=\frac{V^{\prime}}{2} \quad a_{i}^{U}=t \quad q_{i}^{U}=\frac{1}{2} \quad p_{j}^{U}=V^{\prime} \quad q_{j}^{U}=\frac{1}{2} \quad \sum_{j=0,1} I_{i j}^{U}=1 \quad I_{i j}^{U} \neq I_{-i j}^{U} \forall i, j
$$

\section{PROOF: see Appendix.}

We see therefore that, in the unregulated regime, only universal connection and total fragmentation can arise in equilibrium and that they never coexist. ${ }^{27}$ Figure 5 below provides an illustration of the regions where the equilibria described take place, conditionally on the values of $V^{\prime}$ and $V^{\prime \prime}$. The thresholds reported in Proposition 4 are decreasing in the value of contents $\delta$, the first faster than the second. Moreover, the first is decreasing in the extent of complementarity $\gamma$, while the second is independent of it. This implies that a stronger valuation for contents by consumers increases the chances for universal connection and, vice versa, reduces those of total fragmentation. On the other hand, greater content complementarity makes total fragmentation less likely, while not affecting the likelihood of universal connection.

Let us first provide the intuition for the equilibrium entailing total fragmentation. When competition among CPs brings a strong decrease in ad rates (i.e. $V^{\prime \prime}$ is low with respect to $V^{\prime}$ ) and/or availability of content is of little relevance for consumers and/or contents are highly substitutable (i.e. $\delta$ and $\gamma$ are small), ISPs are better off avoiding competition to attract CPs.

\footnotetext{
${ }^{27}$ Indeed, it can be verified that $\left(\frac{V^{\prime}}{2}+\frac{t}{2}-\frac{(3 t+\delta(1+\gamma))^{2}}{18 t}\right)\left(\frac{3 t}{3 t+\delta(1+\gamma)}\right)<V^{\prime}\left(\frac{3 t}{6 t+\delta}\right)$ always holds.
} 
To attract both CPs, ISPs should ask for a very low access fee, renouncing to a large share of profits on the content side of the market, with little benefit on the consumer side. Hence, the equilibrium strategy is to set a high access fee $\frac{V^{\prime}}{2}$. Said differently, per each consumer, an ISP asks for the entire profit made by selling exclusive ad impressions $V^{\prime}$. A CP is willing to pay such a fee if and only if there is no competition to attract advertisers targeting consumers subscribing to the ISP. Consequently, the high fee induces network fragmentation. The most profitable deviation from this equilibrium for an ISP (say, A) is to charge a fee equal to only $V^{\prime \prime}$ per consumer. Given that $B$ maintains the high fee, both CPs join exclusively $A$, i.e. configuration $(0 \mathrm{~A}, 1 \mathrm{~A})$ emerges. By so doing, ISP $A$ becomes more attractive than the rival in the eyes of consumers. However, when $V^{\prime \prime}, \delta$ and $\gamma$ are small, the costs on the content side outweigh the gains on the consumer side and no ISP deviates.

On the contrary, when the presence of multiple CPs on an ISP brings a small decrease in ad rates (i.e. $V^{\prime \prime}$ is high enough with respect to $V^{\prime}$ ) and/or availability of contents is relevant for consumers (i.e. $\delta$ is large), ISPs compete to attract CPs and universal connection arises in equilibrium. Thus, they end up setting a low fee (per consumer) equal to $V^{\prime \prime} q^{-} .{ }^{28}$ While, intuitively, content relevance and complementarity make total fragmentation less likely, complementarity among contents $\gamma$ is irrelevant for universal connection. This is surprising: a priori, one would expect complementarity to increase incentives to attract both contents. The reason this does not happen is that the most profitable deviation from the universal connection equilibrium is for ISP $i$ to raise the fee to a level high enough to induce total fragmentation. By so doing, the ISP gives up on revenues from one $\mathrm{CP}$, at the same time capturing extra profits from the remaining CP through the higher fee. However, when $V^{\prime \prime}$ and $\delta$ are high, the extra profits are too small. Hence, the equilibrium entailing universal connection is sustainable. Moreover, since the network configuration induced by deviating is still symmetric, complementarity of contents is not relevant.

There exists a set of parameters such that no (pure-strategy) equilibrium exists: at least one of the ISPs would like to deviate to a configuration of the network that can not be sustained at equilibrium. The interval is such that

$$
\left(\frac{V^{\prime}}{2}+\frac{t}{2}-\frac{(3 t+\delta(1+\gamma))^{2}}{18 t}\right)\left(\frac{3 t}{3 t+\delta(1+\gamma)}\right)<V^{\prime \prime}<V^{\prime}\left(\frac{3 t}{6 t+\delta}\right)
$$

\footnotetext{
${ }^{28}$ Other equilibria entailing universal connection may arise in which both access fees are smaller than $V^{\prime \prime} q^{-}$, though only if $V^{\prime \prime}$ is strictly larger than $V^{\prime}\left(\frac{3 t}{6 t+\delta}\right)$. We do not elaborate on such equilibria for brevity, since their nature is essentially the same as the one described in Proposition 4: they are the result of tariff competition between ISPs in order to attract CPs. The only difference is that such competition may drive access fees even lower than stated in the Proposition, while all other prices and quantities are invariant.
} 


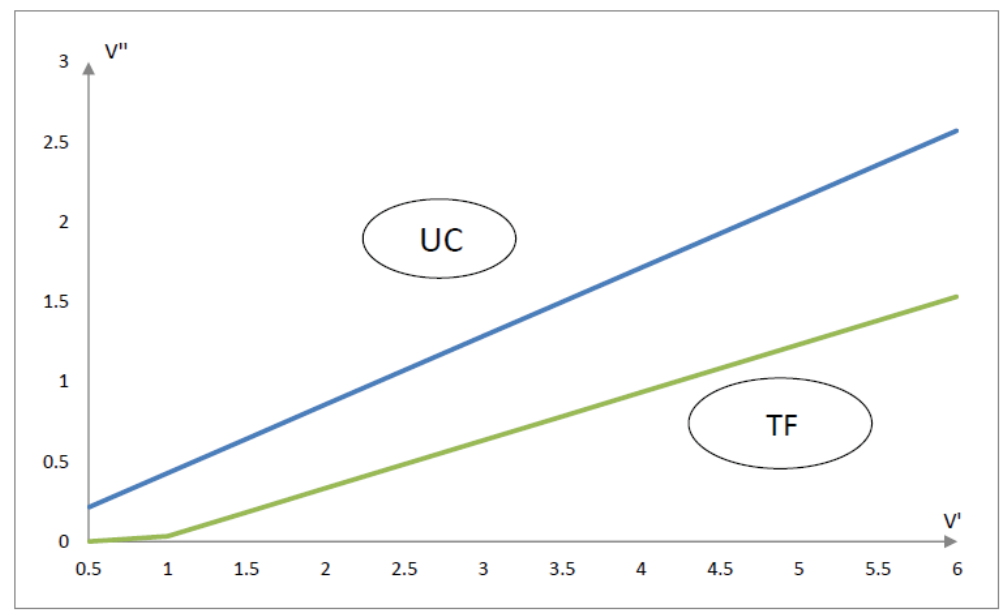

Figure 5: Duopoly, equilibria in the Unregulated regime: the green line represents threshold $\left(\frac{V^{\prime}}{2}+\frac{t}{2}-\frac{(3 t+2 \delta)^{2}}{18 t}\right)\left(\frac{3 t}{3 t+2 \delta}\right)$. The blue line represents threshold $V^{\prime}\left(\frac{3 t}{6 t+\delta}\right)$. UC stands for Universal Connection and TF for Total Fragmentation. Graphs are obtained setting $\delta=0.5$ and $\gamma=1$.

Observe that this interval shrinks as the value of content $\delta$ goes to zero. ${ }^{29}$

As a final remark, we note that there is no equilibrium involving partial fragmentation (where a CP joins two ISPs and the other CP only one). It turns out that there are always profitable deviations from such a configuration for at least one ISP. Take, for example, the case $(0 \mathrm{~A}, 1 \mathrm{AB})$. Two situations are possible. In the first, the value of repeated ad impressions and content attractiveness for consumers are high enough that $B$ (i.e. the ISP with only one $\mathrm{CP})$ prefers to lower its tariff in order to try to have both CPs on board. In the other, $V^{\prime \prime}$ and $\delta$ are low enough that $A$ (i.e. the ISP signing up two CPs) is better off excluding one of them.

\subsection{Comparison of net neutrality and unregulated regimes}

\subsubsection{Network configurations}

Using the results of Propositions 3 and 4, we now proceed to a comparison of network configurations under the two regulatory regimes. ${ }^{30}$ To begin, our results suggest that universal distribution of contents is less likely in the unregulated regime than with net neutrality. First, Propositions 3 and 4 imply that conditions for universal connection to be the unique

\footnotetext{
${ }^{29}$ We do not fully investigate the existence of mixed-strategy equilibria. However, we can prove that when $\left(\frac{V^{\prime}}{2}+\frac{t}{2}-\frac{(3 t+\delta(1+\gamma))^{2}}{18 t}\right)\left(\frac{3 t}{3 t+\delta(1+\gamma)}\right)<V^{\prime \prime}<V^{\prime}\left(\frac{3 t}{6 t+\delta}\right)$, attracting both CPs is a dominated strategyHence, universal connection can never arise as an equilibrium configuration, not even in mixed strategies.

${ }^{30}$ For the sake of clarity, we make this comparison considering parameter values such that pure-strategy equilibria exist under both regimes.
} 


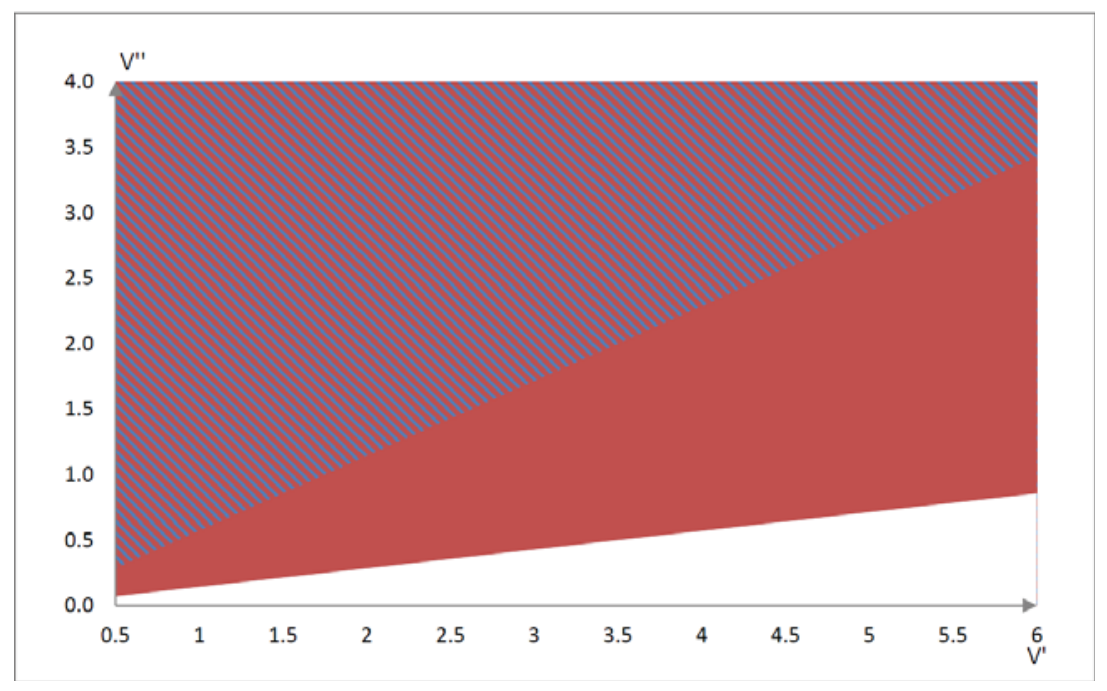

Figure 6: Occurrence of Universal Connection as unique equilibrium under Net Neutrality (red and dashed area) and Unregulated regime (dashed). Graphs are obtained setting $\delta=0.5$ and $\gamma=1$.

equilibrium are always weaker with net neutrality than in its absence. Moreover, universal connection is always an equilibrium with network neutrality, while, in the unregulated regime, it is an equilibrium only when repeated ad impressions do not lose much value compared to a first impression and $\backslash$ or content is highly valuable for consumers. Nevertheless, a change in the regulatory regime does not necessarily imply a change in the network configuration. Indeed, when the impact of competition on advertising profits is small, universal distribution is the only equilibrium, even if net neutrality is removed. See Figure 6 for an illustration.

We have also found that total fragmentation can take place even if net neutrality is maintained. This is the case when competition among CPs strongly reduces their profitability as advertising outlets. Nonetheless, when competition among CPs strongly impacts their profitability, total fragmentation is the unique equilibrium in the unregulated regime, but not with net neutrality. As mentioned, a key difference between the two regimes is that ISPs have a stake in profits made by CPs in the unregulated regime, while they have not under net neutrality. Under the unregulated regime, ISPs take into account that restricting available contents reduces competition on the advertising market. Hence, they may be less interested in distributing all the CPs. This highlights the importance of access fees as strategic variables affecting competition in the online advertising market. In the net neutrality regime, when consumers have a weak valuation for contents and the value of repeated ad impressions is low, each CP could make higher profits by segmenting the market. Yet, free connection to the network means that CPs may not be able to coordinate on a full fragmentation equilibrium. Quite intuitively, in the unregulated regime, access fees act as the missing coordination device. 


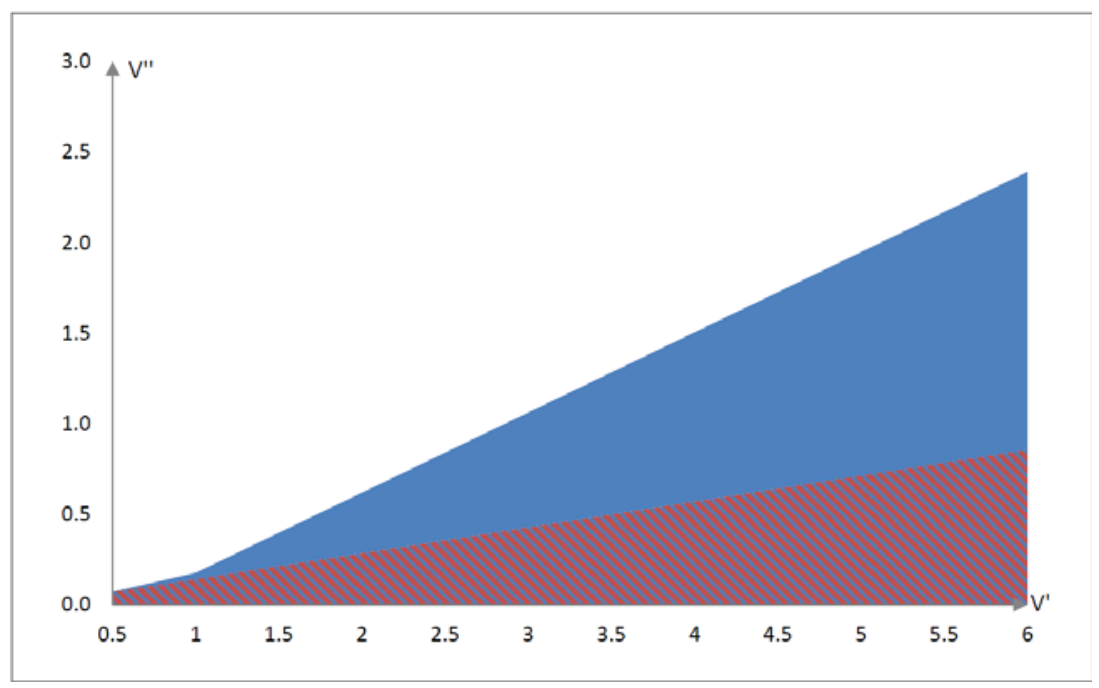

Figure 7: Occurrence of Total fragmentation as unique equilibrium under Net Neutrality (dashed area) and Unregulated regime (blue and dashed). Graphs are obtained setting $\delta=0.5$ and $\gamma=1$.

As a consequence, contrarily to the net neutrality regime, total fragmentation is the unique equilibrium. See Figure 7 for an illustration.

Summing up, the results suggest that, on the one hand, net neutrality certainly helps preserving universal access to online content. On the other hand, the extent to which repeated ad impressions lose value plays an important role in shaping the network configuration, regardless of the regulatory regime. We can thus state the following

Corollary If repeated impressions do not lose much value compared to first impressions (i.e. $V^{\prime}\left(\frac{3 t}{6 t+\delta}\right) \leq V^{\prime \prime}$ ) universal connection is the unique equilibrium configuration, regardless of the regulatory regime. However, when the value of repeated ad impressions is lower, the regulatory regime matters: first, when $V^{\prime}\left(\frac{\delta \gamma}{3 t+\delta \gamma}\right) \leq V^{\prime \prime}<V^{\prime}\left(\frac{3 t}{6 t+\delta}\right)$ universal connection is the unique equilibrium with net neutrality but is impossible in the unregulated regime. Second, when $V^{\prime \prime}<\min \left\{V^{\prime}\left(\frac{\delta \gamma}{3 t+\delta \gamma}\right) ;\left(\frac{V^{\prime}}{2}+\frac{t}{2}-\frac{(3 t+\delta(1+\gamma))^{2}}{18 t}\right)\left(\frac{3 t}{3 t+\delta(1+\gamma)}\right)\right\}$ both total fragmentation and universal connection are equilibria under net neutrality, but only total fragmentation is an equilibrium in the unregulated regime.

\subsubsection{The role of content availability and complementarity}

Propositions 3 and 4 also provide interesting results in terms of the role played by content value and complementarity in shaping network configurations. Interestingly, the extent to which these affect the likelihood of fragmentation changes (radically, in some instances) with 

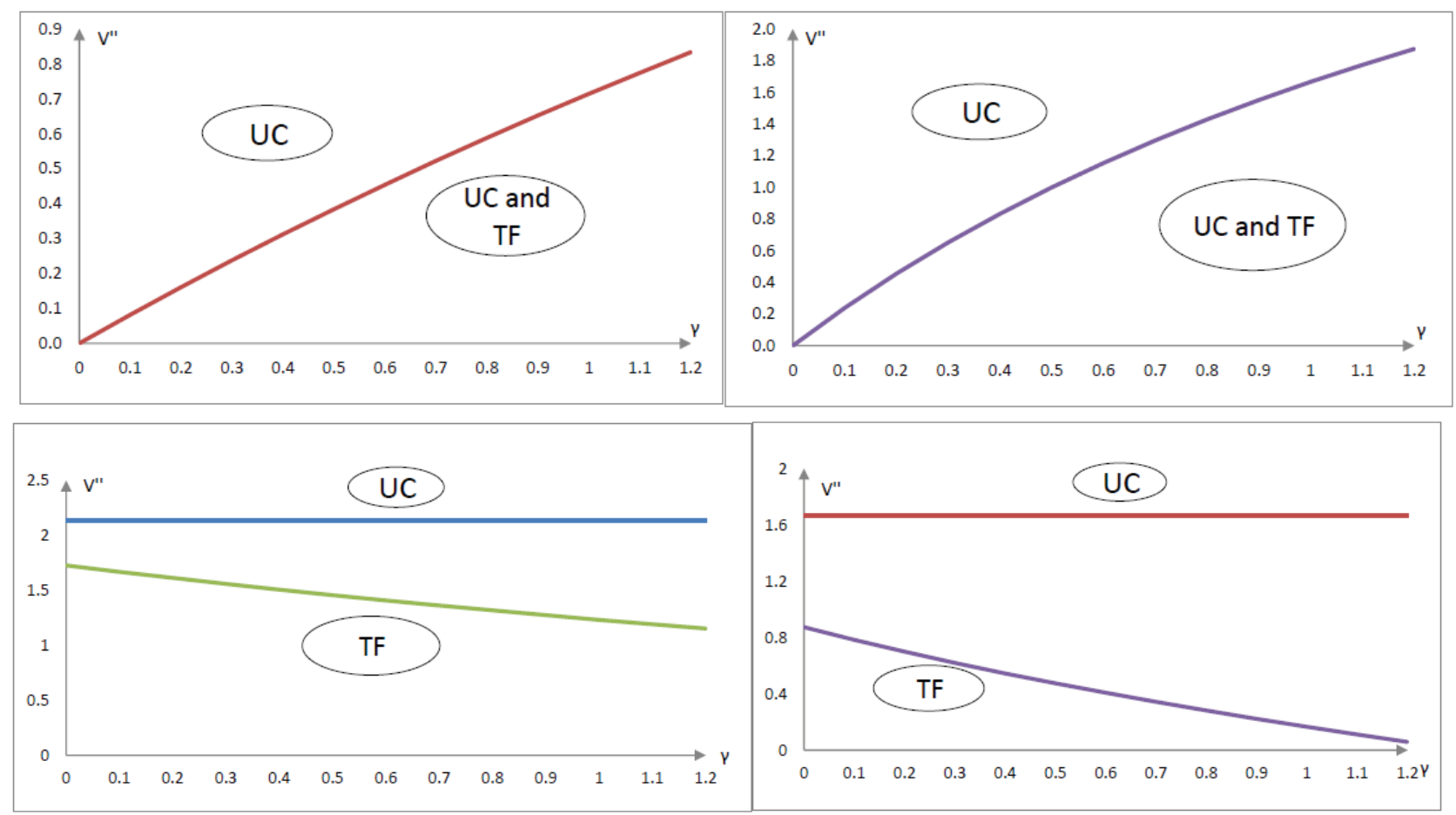

Figure 8: Equilibria in the net neutrality (top) and unregulated (bottom) regimes. The left hand graphs are obtained setting $\delta=0.5$ and the right hand ones setting $\delta=1.5$. We also set $V^{\prime}=5$.

the regulatory regime. First of all, with net neutrality both the absolute valuation for content $\delta$ and complementarity $\gamma$ increase chances for fragmentation and, specularly, reduce those of universal connection. In contrast, greater valuation of content by consumers makes it more likely that universal connection is the unique equilibrium in the unregulated regime, while content complementarity does not play any role. Moreover, in the unregulated regime, the likelihood of total fragmentation decreases with the absolute valuation for content $\delta$ by consumers, as well as with the extent of content complementarity $\gamma$. Figure 8 provides an illustration.

\subsubsection{Welfare comparison}

Finally, we turn to a comparison of welfare under the two regulatory regimes. We first analyze the effects brought by a change in the regulatory regime on each player taken separately. Next, we consider the effects on social welfare.

The equilibrium entailing universal connection is always optimal for consumers. This is because they enjoy all existing contents and transportation costs are minimized. Compared to the total fragmentation scenario, they end up paying the same subscription fee $a=t$, but 
enjoy a larger choice of contents. Indeed, their surplus is $Z+\delta(1+\gamma)-\frac{5}{4} t$ under universal connection and $Z+\delta-\frac{5}{4} t$ with the total fragmentation. From this and the above Corollary, it follows that consumers are weakly better off under net neutrality than under the unregulated regime.

Advertisers are also weakly better off under net neutrality. A change from the universal connection to total fragmentation decreases their total surplus, which is equal to $V^{\prime}-V^{\prime \prime}$ in the former configuration and to zero in the latter. This is because in the total fragmentation case CPs can charge higher prices for ad spaces, extracting all their surplus. On top of this, the number of impressions per ad is reduced, as possibilities for advertisers to reach consumers are restricted. Therefore, like consumers, advertisers are never better off in the unregulated regime, and strictly better off under net neutrality when first ad impressions are much more valuable than repeated ones.

Let us now look at ISPs and CPs. A switch from the net neutrality to the unregulated regime always penalizes CPs while benefiting ISPs. This is true regardless of whether changes in the regulatory regime modify the market configuration or not. Under the unregulated regime, ISPs obtain extra profits from access fees paid by content providers. Moreover, they see no change in profits on the consumer side of the market, since all equilibria are symmetric and total subscription fees collected are invariant. Indeed, the profit of an ISP is always $\pi_{i}^{N}=\frac{t}{2}$ with net neutrality, while it obtains $\pi_{i}^{U}=\frac{t}{2}+2 V^{\prime \prime} q^{-}$with universal connection and $\pi_{i}^{U}=\frac{t}{2}+\frac{V^{\prime}}{2}$ with total fragmentation in the unregulated regime. Each CP gains $\pi_{j}^{N}=V^{\prime \prime}$ with universal connection and $\pi_{j}^{N}=\frac{V^{\prime}}{2}$ with total fragmentation under net neutrality, while it gains, respectively $\pi_{j}^{U}=V^{\prime \prime}-2 V^{\prime \prime} q^{-}$and $\pi_{j}^{U}=0$ in the unregulated regime. Thus, abandoning the net neutrality regime always entails a transfer of profits from CPs to ISPs.

To conclude, let us look at the change in social welfare. It is quite easy to see that the higher the number of connections between ISPs and CPs, the higher the gross surplus generated by both consumers and advertisers. Indeed, as stated in Lemma 1, universal connection is the optimal configuration from a social welfare point of view. Hence, as it maximizes the chances for occurrence of universal connection, net neutrality is the (weakly) optimal regulatory regime. Summing up, we conclude the following

Proposition 5 When repeated ad impression significantly lose value compared to first impressions and content is of small importance for consumers, the unregulated regime reduces consumer, advertiser and social welfare, compared to net neutrality. Otherwise, the regulatory regime is immaterial for consumer, advertiser and social welfare. Finally, ISPs are always better off and CPs always worse off under the unregulated regime than under net neutrality. 
PROOF: the result on total welfare simply follows from Lemma 1, Propositions 3 and 4 . The results on consumer surplus, advertiser surplus, CPs' profits and ISPs' profits are shown in the text.

\section{Conclusions}

We have investigated the implications of net neutrality regulation (intended as a zero-price rule for CPs) for the fragmentation of the Internet. Our analysis has highlighted an important link between fragmentation and the advertising market. In particular, we have shown that, without a zero-price rule, ISPs may use non-discriminatory access fees to rule out universal connection of CPs. The rationale for this is to relax competition among CPs to attract advertisers, recovering their extra profits through the access fees. Our findings suggest that net neutrality makes universal connection more likely. However, abandoning it does not necessarily imply changes in network configuration. First, universal connection can occur also in the unregulated regime, as long as competition among CPs does not reduce the profitability of ad spaces to a large extent. Moreover, when competition has a strong impact, total fragmentation can occur even under net neutrality. Nonetheless, total fragmentation is the unique equilibrium if net neutrality is removed, but not if it is maintained. Finally, since social welfare is maximized with universal connection, the unregulated regime is weakly dominated by net neutrality.

We believe our findings to be an interesting addition to the rapidly-growing literature on the topic. Of course, our analysis has some important limitations. First, we have modeled consumers demand for content as exogenous. However, depending on the availability of content, consumers may vary the intensity of use of the network. This may affect profitability of content providers by creating additional congestion and changing the amount of time consumers spend browsing each content. Second, we have assumed the amount of advertising not to have an effect on consumers demand for content. Third, we have disregarded the issue of quality differentiation and service tiering. While we do not believe including the above features would change our qualitative results, we also recognize that a more thorough investigation of these important issues would be an interesting step for future research.

\section{Appendix}

\section{Proof of Proposition 1}

Looking at the table provided in Lemma 1, we have 
1. Configuration $(0 \mathrm{~A}, 1 \mathrm{~A})$ obtains when

$$
F_{A} \leq V^{\prime \prime} q_{m o n}^{++}
$$

2. $(0 \mathrm{~A}, 1 \mathrm{~N})$ or $(0 \mathrm{~N}, 1 \mathrm{~A})$

$$
V^{\prime \prime} q_{m o n}^{++}<F_{A} \leq V^{\prime} q_{m o n}^{+}
$$

3. $(0 \mathrm{~N}, 1 \mathrm{~N})$

$$
F_{A}>V^{\prime} q_{m o n}^{+}
$$

Setting now all $F_{i}=0$, one easily verifies that only the equilibria described in the claim exist

\section{Proof of Proposition 2}

ISP $A$ can choose whether to provide access to both CPs or to only one CP. Clearly, providing access to no $\mathrm{CP}$ is always a dominated choice. If it provides access to both CPs, ISP $A$ will set the highest access fee CPs are willing to pay given that both join $A$, i.e. $F_{A}=V^{\prime \prime} q_{m o n}^{++}$. In this case, ISP's $A$ profits are

$$
\pi_{A}=2 V^{\prime \prime} q_{m o n}^{++}+\frac{(Z+\delta(1+\gamma))^{2}}{4 t}
$$

If $A$ provides access to only one $\mathrm{CP}$, it will set the highest fee a $\mathrm{CP}$ is willing to pay given that it has exclusive access. set This is $F_{A}=V^{\prime} q_{m o n}^{+} \cdot{ }^{31}$ In this case, ISP's $A$ profits are

$$
\pi_{A}=V^{\prime} q_{m o n}^{+}+\frac{(Z+\delta)^{2}}{4 t}
$$

Hence, ISP $A$ sets $F_{A}=V^{\prime \prime} q_{\text {mon }}^{++}$and induces the network configuration (0A,1A) if

$$
2 V^{\prime \prime} q_{m o n}^{++}+\frac{(Z+\delta(1+\gamma))^{2}}{4 t} \geq V^{\prime} q_{m o n}^{+}+\frac{(Z+\delta)^{2}}{4 t}
$$

that is if

$$
V^{\prime \prime} \geq\left(V^{\prime}\left(\frac{Z+\delta}{2 t}\right)+\frac{(Z+\delta)^{2}}{4 t}-\frac{(Z+\delta(1+\gamma))^{2}}{4 t}\right)\left(\frac{t}{Z+2 \delta}\right)
$$

otherwise, it sets $F_{A}=V^{\prime} q_{m o n}^{+}$and induces the network configuration $\left(j A, j^{\prime} \mathrm{N}\right)$, with $j, j^{\prime} \in$ $\{0,1\} 0 \neq 1$.

\footnotetext{
${ }^{31}$ The configurations $(0 \mathrm{~A}, 1 \mathrm{~N})$ or $(0 \mathrm{~N}, 1 \mathrm{~A})$ can take place if $V^{\prime \prime} q_{m o n}^{++}<V^{\prime} q_{m o n}^{+}$.
} 


\section{Proof of Proposition 3}

Looking at the table provided in Lemma 3, we have

1. Configuration $(0 \mathrm{AB}, 1 \mathrm{AB})$ obtains when

$$
F_{A} \leq V^{\prime \prime} q^{-} \quad F_{B} \leq V^{\prime \prime} q^{-} \quad V^{\prime \prime} \geq F_{A}+F_{B}
$$

2. $(0 \mathrm{AB}, 1 \mathrm{~A})$ and $(0 \mathrm{~A}, 1 \mathrm{AB})$

$$
\begin{array}{cl}
F_{A} \leq V^{\prime \prime} q^{+}-V^{\prime}\left(\frac{1}{2}-q^{-}\right) & V^{\prime \prime} q^{-}<F_{B} \leq V^{\prime} q^{-}-V^{\prime \prime}\left(q^{+++}-q^{+}\right) \\
F_{A}+F_{B} \leq V^{\prime \prime} q^{+}+V^{\prime} q^{-} & F_{B}>F_{A}
\end{array}
$$

and symmetrically for configurations $(0 \mathrm{AB}, 1 \mathrm{~B})$ and $(0 \mathrm{~B}, 1 \mathrm{AB})$, exchanging subscript $A$ with subscript $B$.

3. $(0 \mathrm{~N}, 1 \mathrm{AB})$ and $(0 \mathrm{AB}, 1 \mathrm{~N})$

$$
\begin{aligned}
& V^{\prime \prime} q^{+}<F_{A} \leq V^{\prime} q^{--} \quad V^{\prime \prime} q^{+}<F_{B} \leq V^{\prime} q^{--} \\
& V^{\prime \prime}<F_{A}+F_{B} \leq V^{\prime}
\end{aligned}
$$

4. $(0 \mathrm{~A}, 1 \mathrm{~A})$

$$
\begin{aligned}
F_{A} \leq V^{\prime \prime} q^{+++} \quad F_{B}>V^{\prime} q^{-}-V^{\prime \prime}\left(q^{+++}-q^{+}\right) \\
F_{B}-F_{A}>\frac{V^{\prime}}{2}-V^{\prime \prime} q^{+++}
\end{aligned}
$$

and symmetrically for configuration (0B,1B), exchanging subscript $A$ with subscript $B$.

5. $(0 \mathrm{~A}, 1 \mathrm{~B})$ and $(0 \mathrm{~B}, 1 \mathrm{~A})$

$$
\begin{array}{r}
V^{\prime \prime} q^{+}-V^{\prime}\left(\frac{1}{2}-q^{-}\right)<F_{A} \leq \frac{V^{\prime}}{2} \\
V^{\prime \prime} q^{+}-V^{\prime}\left(\frac{1}{2}-q^{-}\right)<F_{B} \leq \frac{V^{\prime}}{2} \\
-\frac{V^{\prime}}{2}+V^{\prime \prime} q^{+++} \leq F_{A}-F_{B} \leq \frac{V^{\prime}}{2}-V^{\prime \prime} q^{+++}
\end{array}
$$


6. $(0 \mathrm{~N}, 1 \mathrm{~A})$ and $(0 \mathrm{~A}, 1 \mathrm{~N})$

$$
\begin{array}{cc}
V^{\prime \prime} q^{+++}<F_{A} \leq V^{\prime} q^{++} & \frac{V^{\prime}}{2}<F_{B} \\
F_{B}+F_{A}>V^{\prime} q^{-}+V^{\prime \prime} q^{+} & F_{B}>F_{A}
\end{array}
$$

and symmetrically for configuration $(0 \mathrm{~B}, 1 \mathrm{~N})$ and $(0 \mathrm{~N}, 1 \mathrm{~B})$, exchanging subscript $A$ with subscript $B$.

7. $(0 \mathrm{~N}, 1 \mathrm{~N})$

$$
F_{A}>V^{\prime} q^{++} \quad F_{B}>V^{\prime} q^{++} \quad F_{B}+F_{A}>V^{\prime}
$$

Setting now $F_{i}=0 i=A, B$, one easily verifies that only the equilibria described in the claim exist.

\section{Proof of Proposition 4}

In order to determine the sub-game perfect equilibrium, we take each couple of access fees $\left(F_{A}, F_{B}\right)$ and each network configuration associated to it, ${ }^{32}$ and we verify whether there exist profitable deviations for any ISP, given the fee of the rival. If there exists a profitable deviation, the couple of fees considered are not an equilibrium. Note that multiple network configurations could arise for a given couple $\left(F_{A}, F_{B}\right)$. We assume, when evaluating deviations from a given equilibrium, that the ISP anticipates that the configuration to arise is always that which entails the smallest profit. Hence, the set of equilibria we obtain is the largest possible. The Proof is organized in the following way: we consider all possible network configurations and study which couples $\left(F_{A}, F_{B}\right)$ entail them as an equilibrium.

Market configuration $(0 A B, 1 A B)$ From the Proof of Proposition 3, we know that for this configuration to arise we need $F_{i} \leq V^{\prime \prime} q^{-}$for $i=\{A, B\}{ }^{33}$

First, assume that $V^{\prime \prime} \leq \frac{V^{\prime}}{2}$. This implies that $V^{\prime \prime} q^{+}-V^{\prime}\left(\frac{1}{2}-q^{-}\right) \leq V^{\prime \prime} q^{-}$. Suppose both ISPs $i=\{A, B\}$ set $F_{i} \in\left(V^{\prime \prime} q^{+}-V^{\prime}\left(\frac{1}{2}-q^{-}\right) ; V^{\prime \prime} q^{-}\right]$inducing configuration $(0 A B, 1 A B)$ and obtaining $\pi_{i}=2 F_{i}+\frac{t}{2}$. Assume now ISP $A$ deviates by decreasing its fee. It is straightforward that this is never profitable to decrease its fee, since the same network configuration emerges, but $A$ makes less profits. Now, assume that ISP $A$ deviates

\footnotetext{
${ }^{32}$ We have determined the values of the access fees that could give rise to a given market configuration in the Proof of Proposition 3, by checking the incentives of the CPs to accept ISPs offers.

${ }^{33}$ These constraints imply that $F_{A}+F_{B} \leq V^{\prime \prime}$ is always satisfied.
} 
by increasing $F_{A}$. In so doing, $A$ can induce market configuration $(0 B, 1 B)$ or $(0 A, 1 B)$. From the Proof of Proposition 3, since $F_{B} \leq V^{\prime \prime} q^{+++},(0 B, 1 B)$ emerges as long as $F_{A}>$ $\max \left\{V^{\prime} q^{-}-V^{\prime \prime}\left(q^{+++}-q^{+}\right) ; \frac{V^{\prime}}{2}-V^{\prime \prime} q^{+++}+F_{B}\right\}$. Clearly, this deviation never profitable, since $A$ gains only $\pi_{A}=\frac{(3 t-\delta(1+\gamma))^{2}}{18 t}$. Configuration $(0 A, 1 B)$ emerges instead as long as $V^{\prime \prime} q^{+}-V^{\prime}\left(\frac{1}{2}-q^{-}\right)<F_{A} \leq \frac{V^{\prime}}{2}-V^{\prime \prime} q^{+++}+F_{B} \cdot{ }^{34}$ Thus, the most profitable deviation is to set $F_{A}=\frac{V^{\prime}}{2}-V^{\prime \prime} q^{+++}+F_{B}$, obtaining $\pi_{A}=F_{B}+\frac{V^{\prime}}{2}-V^{\prime \prime} q^{+++}+\frac{t}{2}$. It remains to verify whether the deviation is profitable. It is if $F_{B}+\frac{V^{\prime}}{2}-V^{\prime \prime} q^{+++}+\frac{t}{2}>2 F_{A}+\frac{t}{2}$. This depends on parameter values. Of course, a symmetric reasoning can be followed for deviations by ISP $B$. We can conclude that all couple of fees such that $F_{i} \in\left(V^{\prime \prime} q^{+}-V^{\prime}\left(\frac{1}{2}-q^{-}\right) ; V^{\prime \prime} q^{-}\right]$for $i=\{A, B\}$ are an equilibrium inducing network configuration $(0 A B, 1 A B)$ if

$$
V^{\prime \prime} \geq\left(\frac{V^{\prime}}{2}+\max \left\{F_{B}-2 F_{A} ; F_{A}-2 F_{B}\right\}\right) \frac{1}{q^{+++}} \equiv S_{2}\left(F_{A}, F_{B}\right)
$$

Threshold $S_{2}\left(F_{A}, F_{B}\right)$ is smallest when $F_{A}=F_{B}=V^{\prime \prime} q^{-}$. Hence, $F_{A}=F_{B}=V^{\prime \prime} q^{-}$ is the most robust among the equilibrium couples inducing this configuration. Substituting and rearranging, we obtain therefore that

$$
V^{\prime \prime} \geq V^{\prime}\left(\frac{3 t}{6 t+\delta}\right) \equiv S_{2}
$$

is a sufficient condition for the equilibrium inducing $(0 A B, 1 A B)$ to take place. Note also that any access fee $F_{i} \leq V^{\prime \prime} q^{+}-V^{\prime}\left(\frac{1}{2}-q^{-}\right)$for $i=\{A, B\}$ can never arise at equilibrium, since ISP $i$ could increase its fee keeping inducing the network configuration $(0 A B, 1 A B)$ but with higher profits.

Second, assume that $\frac{V^{\prime}}{2}<V^{\prime \prime}<\frac{V^{\prime}(3 t-\delta \gamma)}{3 t+\delta(1-\gamma)}$. This implies that $V^{\prime \prime} q^{-}<\min \left\{V^{\prime \prime} q^{+}-V^{\prime}\left(\frac{1}{2}-q^{-}\right) ; V^{\prime} q^{-}\right.$ Suppose both ISPs $i=\{A, B\}$ fix $F_{i} \leq V^{\prime \prime} q^{-}$inducing the network configuration $(0 A B, 1 A B)$ and obtaining $\pi_{i}=2 F_{i}+\frac{t}{2}$. Assume ISP $A$ deviates by increasing its fee (of course, a similar reasoning can be followed for ISP $B)$. This can induce market configuration $(0 B, 1 A B)$ or $(0 B, 1 B) .{ }^{35}$ If $F_{A}>V^{\prime} q^{-}-V^{\prime \prime}\left(q^{+++}-q^{+}\right), A$ induces market configuration $(0 B, 1 B)$, which is clearly not a profitable deviation. If $A$ sets $F_{A}=V^{\prime} q^{-}-V^{\prime \prime}\left(q^{+++}-q^{+}\right)$, it gives rise to market configuration $(0 B, 1 A B)$ and obtains $\pi_{A}=V^{\prime} q^{-}-V^{\prime \prime}\left(q^{+++}-q^{+}\right)+\frac{(3 t-\delta \gamma)^{2}}{18 t}$. This deviation is profitable if $V^{\prime} q^{-}-V^{\prime \prime}\left(q^{+++}-q^{+}\right)+\frac{(3 t-\delta \gamma)^{2}}{18 t}>2 F_{A}+\frac{t}{2}$. Since this inequality is never verified, the network configuration $(0 A B, 1 A B)$ with access fees $F_{i} \leq V^{\prime \prime} q^{-}$for $i=\{A, B\}$ is always is an equilibrium.

\footnotetext{
${ }^{34}$ Observe that $\min \left\{\frac{V^{\prime}}{2} ; \frac{V^{\prime}}{2}-V^{\prime \prime} q^{+++}+F_{B}\right\}=\frac{V^{\prime}}{2}-V^{\prime \prime} q^{+++}+F_{B}$.

${ }^{35}$ Since in this region $\frac{V^{\prime}}{2}<V^{\prime \prime} q^{+++}$, then the network configuration $(0 B, 1 A)$ can never arise at equilibrium.
} 
Finally, assume that $\frac{V^{\prime}(3 t-\delta \gamma)}{3 t+\delta(1-\gamma)} \leq V^{\prime \prime} \leq V^{\prime}$. This implies that $V^{\prime} q^{-}-V^{\prime \prime}\left(q^{+++}-q^{+}\right) \leq$ $V^{\prime \prime} q^{-}<V^{\prime \prime} q^{+}-V^{\prime}\left(\frac{1}{2}-q^{-}\right)$. Assume both ISPs $i=\{A, B\}$ set $F_{i} \in\left(V^{\prime} q^{-}-V^{\prime \prime}\left(q^{+++}-q^{+}\right) ; V^{\prime \prime} q^{-}\right]$ inducing network configuration $(0 \mathrm{AB}, 1 \mathrm{AB})$ and profits $\pi_{i}=2 F_{i}+\frac{t}{2}$. Assume ISP $A$ deviates by increasing its fee (of course, a similar reasoning can be followed for ISP $B$ ). As ISP $A$ increases its fee, in the worst scenario it looses all CPs, giving rise to market configuration $(0 B, 1 B)$. Since this deviation is never profitable, market configuration $(0 A B, 1 A B)$ with $F_{i} \in\left(V^{\prime} q^{-}-V^{\prime \prime}\left(q^{+++}-q^{+}\right) ; V^{\prime \prime} q^{-}\right]$for $i=\{A, B\}$ is always an equilibrium. Remark that $F_{A}$ and/or $F_{B}$ lower than or equal to $V^{\prime} q^{-}-V^{\prime \prime}\left(q^{+++}-q^{+}\right)$, associated to market configuration $(0 A B, 1 A B)$ can never arise at equilibrium, since, by increasing its fee, an ISP can induce the same market configuration and earn more profits.

Market configuration $\left(0 i, 1 i i^{\prime}\right)$, with $i, i^{\prime} \in\{A, B\}$ and $i \neq i^{\prime}$ (and symmetric) For concreteness, let us focus on configuration $(0 A, 1 A B)$. From the Proof of Proposition 3 , we know that this market configuration is feasible if $F_{A} \leq V^{\prime \prime} q^{+}-V^{\prime}\left(\frac{1}{2}-q^{-}\right), V^{\prime \prime} q^{-}<$ $F_{B} \leq V^{\prime} q^{-}-V^{\prime \prime}\left(q^{+++}-q^{+}\right)$and $F_{B}>F_{A}$. Note that condition $F_{A}+F_{B} \leq V^{\prime \prime} q^{+}+V^{\prime} q^{-}$ is always verified given that the previous inequalities hold. The only fees that can sustain this configuration of the network at equilibrium are $F_{A}=V^{\prime \prime} q^{+}-V^{\prime}\left(\frac{1}{2}-q^{-}\right)$and $F_{B}=V^{\prime} q^{-}-V^{\prime \prime}\left(q^{+++}-q^{+}\right)$. Indeed, lower access fees (still within the above intervals) are dominated, since an ISP can always increase its fee, induce the same configuration and gain strictly higher profits. ISPs' profits are $\pi_{A}=2\left(V^{\prime \prime} q^{+}-V^{\prime}\left(\frac{1}{2}-q^{-}\right)\right)+\frac{(3 t+\delta \gamma)^{2}}{18 t}$ and $\pi_{B}=V^{\prime} q^{-}-V^{\prime \prime}\left(q^{+++}-q^{+}\right)+\frac{(3 t-\delta \gamma)^{2}}{18 t}$. Let us now describe conditions for this equilibrium to emerge. First, assume that $V^{\prime \prime} \leq \frac{V^{\prime}}{2}$, which implies $V^{\prime \prime} q^{+}-V^{\prime}\left(\frac{1}{2}-q^{-}\right) \leq V^{\prime \prime} q^{-}$. Assume that ISP $A$ deviates by decreasing $F_{A}$. This cannot be a profitable deviation for $A$, since it induces the same network configuration, but gains less profits. Now, assume ISP $A$ deviates from this point by increasing $F_{A}$. By so doing, ISP $A$ can either induce market configuration $(0 B, 1 B)$ or $(0 A, 1 B)$. If $F_{A}>\frac{V^{\prime}}{2}-V^{\prime \prime} q^{+++}+F_{B}=\frac{V^{\prime}}{2}+V^{\prime} q^{-}+$ $V^{\prime \prime} q^{+}-2 V^{\prime \prime} q^{+++}$configuration $(0 B, 1 B)$ arises, and this is not a profitable deviation for $A$. Instead, if ISP $A$ sets $F_{A}=\min \left\{\frac{V^{\prime}}{2} ; \frac{V^{\prime}}{2}-V^{\prime \prime} q^{+++}+F_{B}\right\}$, it gives rise to market configuration $(0 A, 1 B)$ and obtains profits $\pi_{A}=\min \left\{\frac{V^{\prime}}{2} ; \frac{V^{\prime}}{2}+V^{\prime} q^{-}+V^{\prime \prime} q^{+}-2 V^{\prime \prime} q^{+++}\right\}+\frac{t}{2}$. We now need to distinguish among two subcases. If $V^{\prime} q^{-}-V^{\prime \prime}\left(q^{+++}-q^{+}\right) \geq V^{\prime \prime} q^{+++}$, $\min \left\{\frac{V^{\prime}}{2} ; \frac{V^{\prime}}{2}+V^{\prime} q^{-}+V^{\prime \prime} q^{+}-2 V^{\prime \prime} q^{+++}\right\}=\frac{V^{\prime}}{2}$. Otherwise, $\min \left\{\frac{V^{\prime}}{2} ; \frac{V^{\prime}}{2}+V^{\prime} q^{-}+V^{\prime \prime} q^{+}-2 V^{\prime \prime} q^{+++}\right\}=$ $\frac{V^{\prime}}{2}+V^{\prime} q^{-}+V^{\prime \prime} q^{+}-2 V^{\prime \prime} q^{+++}$. Thus, if $V^{\prime} q^{-}-V^{\prime \prime}\left(q^{+++}-q^{+}\right) \geq V^{\prime \prime} q^{+++}$, the deviation is profitable for ISP $\frac{V^{\prime}}{2}+\frac{t}{2}>2\left(V^{\prime \prime} q^{+}-V^{\prime}\left(\frac{1}{2}-q^{-}\right)\right)+\frac{(3 t+\delta \gamma)^{2}}{18 t}$, that is 


$$
V^{\prime \prime}<\left(V^{\prime}\left(1+\frac{\delta \gamma}{6 t}\right)+\frac{t}{2}-\frac{(3 t+\delta \gamma)^{2}}{18 t}\right)\left(\frac{6 t}{9 t+2 \delta+3 \delta \gamma}\right) \equiv S_{4}
$$

If instead $V^{\prime} q^{-}-V^{\prime \prime}\left(q^{+++}-q^{+}\right)<V^{\prime \prime} q^{+++}$, the deviation is profitable if

$$
V^{\prime \prime}<\left(V^{\prime}\left(\frac{1}{2}+\frac{2 \delta \gamma}{6 t}\right)+\frac{t}{2}-\frac{(3 t+\delta \gamma)^{2}}{18 t}\right)\left(\frac{3 t}{3 t+\delta \gamma}\right) \equiv S_{6}
$$

Consider now possible deviations by ISP $B$. Suppose it does so by decreasing $F_{B}$. By setting $F_{B}=V^{\prime \prime} q^{-}$, B induces market configuration $(0 A B, 1 A B)$ and obtains profits equal to $\pi_{B}=$ $2 V^{\prime \prime} q^{-}+\frac{t}{2}$. This is profitable if $2 V^{\prime \prime} q^{-}+\frac{t}{2}>V^{\prime} q^{-}-V^{\prime \prime}\left(q^{+++}-q^{+}\right)+\frac{(3 t-\gamma \delta)^{2}}{18 t}$, that is if

$$
V^{\prime \prime}>\left(V^{\prime}\left(\frac{1}{2}-\frac{\delta \gamma}{6 t}\right)-\frac{t}{2}+\frac{(3 t-\delta \gamma)^{2}}{18 t}\right)\left(\frac{6 t}{6 t+\delta-2 \delta \gamma}\right) \equiv S_{5}
$$

Suppose now $B$ deviates by increasing $F_{B}$ : this can only induce market configuration $(0 A, 1 A)$, which is never profitable. It follows that the couple of fees $F_{A}=V^{\prime \prime} q^{+}-V^{\prime}\left(\frac{1}{2}-q^{-}\right)$and $F_{B}=V^{\prime} q^{-}-V^{\prime \prime}\left(q^{+++}-q^{+}\right)$, inducing configuration $(0 A, 1 A B)$ are an equilibrium only if $V^{\prime \prime} \geq S_{4}$ and $V^{\prime \prime} \leq S_{5}$ for $V^{\prime} q^{-}-V^{\prime \prime}\left(q^{+++}-q^{+}\right) \geq V^{\prime \prime} q^{+++}$and if $V^{\prime \prime} \geq S_{6}$ and $V^{\prime \prime} \leq S_{5}$ for $V^{\prime} q^{-}-V^{\prime \prime}\left(q^{+++}-q^{+}\right)<V^{\prime \prime} q^{+++}$. It can be shown that this region is empty. Therefore, network configurations $(0 A, 1 A B)$ and $(0 A B, 1 A)$, as well as all the symmetric, never arise at equilibrium.

Second, suppose that $\frac{V^{\prime}}{2}<V^{\prime \prime}<\frac{V^{\prime}(3 t-\delta \gamma)}{3 t+\delta(1-\gamma)}$, which implies $V^{\prime \prime} q^{-}<\min \left\{V^{\prime \prime} q^{+}-V^{\prime}\left(\frac{1}{2}-q^{-}\right) ; V^{\prime} q^{-}-V\right.$ Assume ISP $B$ deviates by decreasing $F_{B}$ to $V^{\prime \prime} q^{+}-V^{\prime}\left(\frac{1}{2}-q^{-}\right)-\varepsilon$, where $\varepsilon>0$ and small. This induces market configuration $(0 B, 1 A B)$ or $(0 A B, 1 B)$ so $B$ earns $\pi_{B}=2\left(V^{\prime \prime} q^{+}-V^{\prime}\left(\frac{1}{2}-q^{-}\right)-\varepsilon\right)+$ $\frac{(3 t+\delta \gamma)^{2}}{18 t}$. Since it is always verified that $2\left(V^{\prime \prime} q^{+}-V^{\prime}\left(\frac{1}{2}-q^{-}\right)-\varepsilon\right)+\frac{(3 t+\delta \gamma)^{2}}{18 t}>V^{\prime} q^{-}-$ $V^{\prime \prime}\left(q^{+++}-q^{+}\right)+\frac{(3 t-\delta \gamma)^{2}}{18 t}$, this deviation is always profitable. It follows that configurations $(0 A, 1 A B)$ and $(0 A B, 1 A)$ and their symmetric are never equilibria.

Finally, assume $\frac{V^{\prime}(3 t-\delta \gamma)}{3 t+\delta(1-\gamma)} \leq V^{\prime \prime} \leq V^{\prime}$, which implies $V^{\prime} q^{-}-V^{\prime \prime}\left(q^{+++}-q^{+}\right) \leq V^{\prime \prime} q^{-}<$ $V^{\prime \prime} q^{+}-V^{\prime}\left(\frac{1}{2}-q^{-}\right)$. Since $V^{\prime} q^{-}-V^{\prime \prime}\left(q^{+++}-q^{+}\right)<V^{\prime \prime} q^{+}-V^{\prime}\left(\frac{1}{2}-q^{-}\right)$constraint $F_{B}>F_{A}$ is always violated. Hence, configurations $(0 A, 1 A B)$ and $(0 A B, 1 A)$ and their symmetric are never equilibria.

Market configuration $\left(0 i, 1 i^{\prime}\right)$, with $i, i^{\prime} \in\{A, B\}$ and $i \neq i^{\prime}$ (and symmetric) Following the Proof of Proposition 3, we know that this market configuration is feasible if the following constraints hold: $V^{\prime \prime} q^{+}-V^{\prime}\left(\frac{1}{2}-q^{-}\right)<F_{i} \leq \frac{V^{\prime}}{2} i=A, B$ and $-\frac{V^{\prime}}{2}+V^{\prime \prime} q^{+++} \leq$ $F_{i}-F_{i^{\prime}} \leq \frac{V^{\prime}}{2}-V^{\prime \prime} q^{+++} i, i^{\prime} \in\{A, B\}, i \neq i^{\prime}$. Among all couples of fees that respect the above conditions, the only sustainable at equilibrium are $F_{A}=F_{B}=\frac{V^{\prime}}{2}$. Starting from any 
other couple, an ISP could always marginally increase its fee, induce the same configuration and gain higher profits. Consequently, $\pi_{A}=\pi_{B}=\frac{V^{\prime}}{2}+\frac{t}{2}$.

First, assume that $V^{\prime \prime} \leq \frac{V^{\prime}}{2}$, which implies $V^{\prime \prime} q^{+}-V^{\prime}\left(\frac{1}{2}-q^{-}\right) \leq V^{\prime \prime} q^{-}$. Suppose ISP $A$ deviates by decreasing its fee to the largest one inducing configuration $(0 A, 1 A)$ (given that $\left.F_{B}=\frac{V^{\prime}}{2}\right)$, i.e. to $\min \left\{F_{i^{\prime}}-\frac{V^{\prime}}{2}+V^{\prime \prime} q^{+++}-\varepsilon ; V^{\prime \prime} q^{+++}\right\}=V^{\prime \prime} q^{+++}-\varepsilon$, where $\varepsilon>0$ and small. By so doing, $A$ earns profits $\pi_{A}=2\left(V^{\prime \prime} q^{+++}-\varepsilon\right)+\frac{(3 t+\delta(1+\gamma))^{2}}{18 t}$. The deviation is profitable if $2\left(V^{\prime \prime} q^{+++}-\varepsilon\right)+\frac{(3 t+\delta(1+\gamma))^{2}}{18 t}>\frac{V^{\prime}}{2}+\frac{t}{2}$, that is if

$$
V^{\prime \prime}>\left(\frac{V^{\prime}}{2}-\frac{(3 t+\delta(1+\gamma))^{2}}{18 t}+\frac{t}{2}\right)\left(\frac{3 t}{3 t+\delta(1+\gamma)}\right) \equiv S_{1}
$$

Suppose now $A$ deviates by increasing its fee. Obviously, it can only lose the only CP that it has on board. Hence, this deviation is not profitable. Summing up, the market configuration $\left(0 i, 1 i^{\prime}\right)$ with $F_{A}=F_{B}=\frac{V^{\prime}}{2}$ is an equilibrium only if no ISP finds profitable to deviate, that is if $V^{\prime \prime} \leq S_{1}$.

Second, suppose now that $\frac{V^{\prime}}{2}<V^{\prime \prime}<\frac{V^{\prime}(3 t-\delta \gamma)}{3 t+\delta(1-\gamma)}$, which implies $V^{\prime \prime} q^{-}<\min \left\{V^{\prime \prime} q^{+}-V^{\prime}\left(\frac{1}{2}-q^{-}\right) ; V^{\prime} q\right.$ Suppose ISP $A$ deviates decreasing its fee. As before, by fixing its fee equal to $\min \left\{F_{B}-\frac{V^{\prime}}{2}+V^{\prime \prime} q^{+++}-\right.$ $V^{\prime \prime} q^{+++}-\varepsilon$ it can induce market configuration $(0 A, 1 A)$ and earn $\pi_{A}=2\left(V^{\prime \prime} q^{+++}-\varepsilon\right)+$ $\frac{(3 t+\delta(1+\gamma))^{2}}{18 t}$. As in the above case, the deviation is profitable only if $V^{\prime \prime}>S_{1}$. Since we verify that $V^{\prime \prime}>S_{1}$, then configuration $\left(0 i, 1 i^{\prime}\right)$ never arises at equilibrium in this region.

Finally, assume $\frac{V^{\prime}(3 t-\delta \gamma)}{3 t+\delta(1-\gamma)} \leq V^{\prime \prime} \leq V^{\prime}$, which implies $V^{\prime} q^{-}-V^{\prime \prime}\left(q^{+++}-q^{+}\right) \leq V^{\prime \prime} q^{-}<$ $V^{\prime \prime} q^{+}-V^{\prime}\left(\frac{1}{2}-q^{-}\right)$. Since $V^{\prime \prime} q^{+++}>\frac{V^{\prime}}{2}$, constraint $-\frac{V^{\prime}}{2}+V^{\prime \prime} q^{+++} \leq F_{i}-F_{i^{\prime}} \leq \frac{V^{\prime}}{2}-V^{\prime \prime} q^{+++}$ is always violated and this configuration is never feasible.

Market configuration (0N, $\left.1 i i^{\prime}\right)$, with $i, i^{\prime} \in\{A, B\}$ and $i \neq i^{\prime}$ (and symmetric) Following the Proof of Proposition 3, this market configuration is feasible if $V^{\prime \prime} q^{+}<F_{A} \leq$ $V^{\prime} q^{--}$. If this constraint is verified, then also the constraint $V^{\prime \prime}<F_{A}+F_{B} \leq V^{\prime}$ is always verified. It can be observed that the region where this configuration is feasible is a subset of the region where $\left(0 i, 1 i^{\prime}\right)$ is feasible. For a given access fee, ISP $i$ earns the same profits under configuration $\left(0 \mathrm{~N}, 1 i i^{\prime}\right)$ or $\left(0 i i^{\prime}, 1 \mathrm{~N}\right)$ and $\left(0 i, 1 i^{\prime}\right)$ (since the profits on the consumers' side in both cases are $\frac{t}{2}$ ). Hence, an ISP can always deviate from this equilibrium by raising its fee, so as to induce $\left(0 i, 1 i^{\prime}\right)$. In this way, it earns higher profits.

Market configuration $(\mathbf{0} i, \mathbf{1} i),(\mathbf{0 N}, \mathbf{1} i),(\mathbf{0 N}, \mathbf{1 N})$ with $i \in\{A, B\}$ (and symmetric) An ISP always finds profitable to deviate from this configuration. Indeed, it is straightforward that by a small reduction in its fee, an ISP can increase its revenues. 


\section{References}

[1] Ambrus, A., Calvano, E. and Reisinger, M. (2012), Either or both competition: a "twosided" theory of advertising with overlapping viewerships, Working Paper available at http://public.econ.duke.edu/ aa231/AAEM_8b.pdf

[2] Ambrus, A. and Reisinger, M. (2006), Exclusive vs Overlapping Viewers in Media Markets, Working Paper, Harvard University.

[3] Anderson, S.P. and Coate, S. (2005), Market Provision of Broadcasting: A Welfare Analysis, Review of Economic Studies, 72, 947-972.

[4] Anderson, S.P., Foros, O. and Kind, H.J. (2011), Competition for advertisers in media markets, Working Paper available at http://www.econ.vt.edu/seminars/seminarpapers/2011/simonanderson04222011.pdf.

[5] Athey, S., Calvano, E. and Gans, J.S. (2011), The Impact of the Internet on Advertising Markets for News Media, Working Paper available at http://kuznets.harvard.edu/ athey/papers/Impact-Internet-Adv-Mkts-4-27-12.pdf

[6] Bourreau, M., Kourandi, F. and Valletti, T.M. (2012), Net Neutrality with Competing Internet Platforms, Working Paper available at http://papers.ssrn.com/sol3/papers.cfm?abstract_id=2061571.

[7] Calvano, E. and Jullien B. (2012), Issues in online advertising and competition policy: a two-sided market perspective, Recent Advances in the Analysis of Competition Policy and Regulation, edited by J.E. Harrington and Y. Katsoulacos, Edward Elgar Publishing.

[8] Choi, J.P., Jeon, D.S. and Kim, B.C. (2012), Internet Interconnection and Network Neutrality, TSE Working Paper Series, 12-355.

[9] Choi, J.P. and Kim, B.C. (2010), Net neutrality and investment incentives, The RAND Journal of Economics, 41, 446-471.

[10] Economides, N. and Hermalin, B. (2012), The Economics of Network Neutrality, RAND Journal of Economics, 43, 602-629.

[11] Economides, N. and Tag J. (2012), Network neutrality on the Internet: A two-sided market analysis, Information Economics and Policy, 24, 91-104. 
[12] Federal Communications Commission (2005), New Principles to Preserve and Promote the Open and Interconnected Nature of Public Internet, Retrieved 2006-07-07.

[13] Harsanyi, J.C. (1995), A New Theory of Equilibrium Selection for Games with Complete Information, Games and Economic Behavior, 8, 91-122.

[14] Hermalin B.E. and Katz, M.L. (2007), The economics of product-line restrictions with an application to the network neutrality debate, Information Economics and Policy, 19, 215-248.

[15] Kourandi, F., Krämer, J. and Valletti, T.M. (2012), Net Neutrality, Exclusivity Contracts and Internet Fragmentation, Mimeo.

[16] Krämer, J. and Wiewiorra, L. (2012), Network Neutrality and Congestion Sensitive Content Providers: Implications for Content Variety, Broadband Investment, and Regulation, Information Systems Research, 23, 303-1321.

[17] Krämer J., L. Wiewiorra and C. Weinhardt (2012), Net neutrality: A progress report, Telecommunications Policy, forthcoming.

[18] Lee, R.S. and T. Wu (2009), Subsidizing creativity through network design: Zeropricing and net neutrality, Journal of Economic Perspectives, 23, 61-76.

[19] Musacchio, J., G. Schwartz, and J. Walrand (2009), A two-sided market analysis of provider investment incentives with an application to the net-neutrality issue, Review of Network Economics, 8, 22-39.

[20] Schuett, F. (2010), Network Neutrality: A Survey of the Economic Literature, Review of Network Economics, 9, Article 1.

[21] Taylor G. (2012), "Attention Retention Targeted Advertising and the Provision of Media Content". Mimeo

[22] Werbach K. (2009), The Centripetal Network: How the Internet Holds Itself Together, and the Forces Tearing It Apart. UC Davis Law Review, Vol. 42, p. 343, 2009. 


\section{Author contacts:}

\section{Anna D’Annunzio}

Università degli Studi di Roma "La Sapienza"- DIAG

Via Ariosto 25

00185, Rome

Email :dannunzio@dis.uniroma1.it

\section{Antonio Russo}

Robert Schuman Centre for Advanced Studies, EUI

Villa La Fonte

Via delle Fontanelle 10

50014 S. Domenico di Fiesole (FI)

Italy

Email: antonio.russo@eui.eu 